\title{
A Modified TODIM Based on Compromise Distance for MAGDM with $q$-Rung Orthopair Trapezoidal Fuzzy Numbers
}

\author{
Benting Wan $\mathbb{D D}^{1}{ }^{1}$ Juelin Huang, ${ }^{1}$ and Xiaolu Zhang $\mathbb{D D}^{2}$ \\ ${ }^{1}$ School of Software and Internet of Things Engineering, Jiangxi University of Finance and Economics, Nanchang 330013, China \\ ${ }^{2}$ The Collaborative Innovation Center, Jiangxi University of Finance and Economics, Nanchang 330013, China \\ Correspondence should be addressed to Xiaolu Zhang; xiaolu_jy@163.com
}

Received 20 August 2021; Revised 10 October 2021; Accepted 11 October 2021; Published 9 November 2021

Academic Editor: Zhen Zhang

Copyright (C) 2021 Benting Wan et al. This is an open access article distributed under the Creative Commons Attribution License, which permits unrestricted use, distribution, and reproduction in any medium, provided the original work is properly cited.

The $q$-rung orthopair fuzzy number ( $q$-ROFN) has been recently developed by Yager and has been widely applied in handling reallife decision-making problems. To enhance its usefulness in dealing with complex practical issues, this paper first proposes the new concept of $q$-rung orthopair trapezoidal fuzzy numbers ( $q$-ROTrFNs) which is a new and useful extension of $q$-ROFNs. Then, we investigate the operation of $q$-ROTrFNs and develop a new ranking method for $q$-ROTrFNs. We also propose a new $q$-rung orthopair trapezoidal fuzzy Hamming distance measure. More important, we develop a useful $q$-rung orthopair trapezoidal fuzzy modified TODIM group decision-making method. In this method, a new $q$-rung orthopair trapezoidal fuzzy weighted aggregating ( $q$-ROTrFWA) operator is developed to integrate individual decision matrices into the collective decision matrix, and a $q$-rung orthopair trapezoidal fuzzy distance measure-based compromise approach is proposed to determine the relative dominance degree of alternatives. It is worth to mention that the modified TODIM method not only expands the freedom of decision makers but also allows decision makers to choose the appropriate risk preference parameter. Finally, a case study on health management of hypertensive patients is conducted to demonstrate the feasibility of the modified TODIM group decision-making method, and the developed method is further verified by comparison analysis with the existing methods and sensitive analysis of different parameters.

\section{Introduction}

Due to the complexity and uncertainty in real-life decisionmaking processes, it is difficult for experts to timely and accurately provide the estimated results in form of exact real numbers. To this end, Zadeh [1] proposed fuzzy set theory and advised experts to use membership functions instead of exact real (nonfuzzy) numbers to describe the estimated results in practical multiattribute decision-making (MADM) problems. Atanassov [2] further proposed the concept of intuitionistic fuzzy sets (IFSs) which include the degree of membership, the degree of nonmembership degree, and the hesitation degree. One important constraint is that the membership degree $(u)$ and nonmembership degree $(v)$ in IFS satisfy $u+v \leq 1$. In order to make IFSs suitable for more complex decision-making environment, Yager [3] proposed the concept of Pythagorean fuzzy set (PFS) in which the square sum of the membership and nonmemberships is less than one. Zhang and $\mathrm{Xu}$ [4] originally provided the mathematical expression form of PFSs and built the basic theoretical framework of PFSs. Subsequently, Zhang [5] extended PFSs into interval-valued fuzzy environment and developed interval-valued PFSs. Despite the usefulness of Pythagorean fuzzy theory $[6,7]$, practical applications have shown that PFSs still have some limitations. Therefore, Yager [8] recently developed the concept of generalized orthopair fuzzy set (is also called $q$-rung orthopair fuzzy set and is known simply as $q$-ROFS) in which $u^{q}+v^{q} \leq 1(q \geq 1)$. The proposed q-ROFSs well incorporate the advantages of IFSs and PFSs and describe a wider range of information within different values of the parameter $q$. The $q$-ROFSs have been a research hotspot in recent years, and many excellent research results have been achieved, for example, the q-rung orthopair fuzzy approximate reasoning method [9], integrals 
and differentials of $q$-rung orthopair fuzzy functions [10-12], various $q$-rung orthopair fuzzy aggregation operators [13-16], q-rung orthopair fuzzy information measures $[17,18]$, and different $q$-rung orthopair fuzzy decisionmaking methods $[19,20]$ have been developed.

Furthermore, with the development of fuzzy sets, the application of trapezoidal fuzzy numbers has been popularized. The trapezoidal fuzzy function is increased and maintained for a period of time and then decreased. Its maximum membership degree can be maintained for a period of time so that it can fit more realistic scenes. If the uncertainty of the alternative presents such a trend and it is presented in the form of interval, the trapezoidal fuzzy number is the most suitable for describing it. However, due to the complexity of the real-life decision-making environment, in some situations, the sum of the membership degree and the nonmembership degree are sometimes greater than 1, and the trapezoidal fuzzy number is not enough to adapt to the realistic decision-making situations. Motivated by the ideas of $q$-ROFS [8] and trapezoidal fuzzy numbers, one important work of this paper is to introduce the concept of $q$-rung orthopair trapezoidal fuzzy numbers ( $q$-ROTrFNs). We will investigate the operation of $q$ ROTrFNs and propose a ranking method for comparing $q$ ROTrFNs. We also propose a new $q$-rung orthopair trapezoidal fuzzy Hamming distance measure. As a useful extension of $q$-ROFS, the $q$-ROTrFNs have also wide application fields, especially in decision-making fields. In order to well handle complex practical MADM or group MADM (MAGDM) problems under $q$-ROTrFNs environment, it is necessary to develop correspondingly effective decision-making methods. TODIM (interactive and multicriteria decision-making) is a multicriteria method proposed by Gomes and Lima [21] on the basis of prospect theory. The biggest advantage of this method is that it can take into account the psychological behavior of decision makers and can well solve decision-making problems with risk characteristics. Gomes et al. [22] developed a Choquet integralbased TODIM method to deal with attributes' interactions. To deal with complex practical MADM issues, many new extensions of TODIM methods, for instance, the hybrid TODIM method [23], the intuitionistic fuzzy TODIM method [24], the hesitant fuzzy TODIM method [25], the interval type-2 fuzzy TODIM method [26], the Pythagorean fuzzy TODIM approach [27], the double hierarchy hesitant fuzzy linguistic entropy-based TODIM approach [28], unbalanced hesitant fuzzy linguistic TODIM approach [29], linguistic distribution TODIM method [30], and hesitant fuzzy linguistic TODIM method [31] have been developed. Tian et al. [32] conducted a detail state-of the-art survey for the development of TODIM with different types of fuzzy sets. Although these studies are able to effectively deal with MAGDM problems, it is indispensable to further extend the TODIM method. On the one hand, the classical TODIM method distinguished decision data by subtraction, which cannot solve the MAGDM problem in the q-rung orthopair trapezoidal fuzzy environment. On the other hand, the modified TODIM method in this paper uses the compromise distance to capture the difference between $q$-ROTrFNs based on the compromise theory. Not only can the distance parameter combined with risk parameter of TODIM reflect the attitude of decision makers facing risks but also the integrity of the information will be ensured to a large extent so that the result is more in line with the actual situation.

Therefore, we attempt to develop a modified TODIM group decision-making method based on $q$-ROTrFNs data. We first develop a new $q$-rung orthopair trapezoidal fuzzy weighted average aggregating ( $q$-ROTrFWA) operator to integrate the individual decision matrix into the collective decision matrix. On the basis of the positive-ideal Hamming distance and the negative-ideal Hamming distance, a new q-rung orthopair trapezoidal fuzzy compromise distance measure is developed for calculating the relative dominance degrees of alternatives. This paper is organized as follows. Section 2 briefly reviews the basic concepts of $q$-ROFNs and Pythagorean trapezoidal fuzzy numbers (PTrFNs). In Section 3, the concept of $q$-ROTrFNs is defined, the operation of $q$-ROTrFNs, the $q$-ROTrFWA operator, and the new the ranking method, and Hamming distance are developed for $q$-ROTrFNs. In Section 4 , the modified TODIM method is proposed, and the group decision-making method based on $q$-ROTrFNs is also given. In Section 5, an example is given to demonstrate the feasibility of the modified TODIM group decision-making method, and Section 6 summarizes this article.

\section{Preliminaries}

This section describes some basic knowledge involved in this paper, including the definition and operation of $q$-ROFS and the definition and operation of PTrFNs.

Definition 1 (see [8]). Let $X$ be the universe of discourse; $A=\left\{x, u_{A}(x), v_{A}(x) \mid x \in X\right\}$ is $q$-ROFS on the universe $X$, the degree of membership $u_{A}: X \longrightarrow[0,1]$, and the degree of nonmembership $v_{A}: X \longrightarrow[0,1]$, and they are satisfied by the following equation.

$$
0 \leq\left(u_{A}(x)\right)^{q}+\left(v_{A}(x)\right)^{q} \leq 1,
$$

where $q \geq 1$, and the degree of hesitation $\pi_{A}(x)$ can be expressed as

$$
\pi_{A}(x)=\sqrt[q]{1-\left(u_{A}(x)\right)^{q}+\left(v_{A}(x)\right)^{q}}, \quad(q \geq 1) .
$$

Definition 2 (see [8]). Let $a=\left(u_{a}, v_{a}\right)$ and $\beta=\left(u_{\beta}, v_{\beta}\right)$ be two $q$-ROFNs; $\gamma>0$ is a random number; then, the following equations hold:

$$
\begin{aligned}
a \oplus \beta & =\left(\left(u_{a}^{q}+u_{\beta}^{q}-u_{a}^{q} u_{\beta}^{q}\right)^{1 / q}, v_{a} v_{\beta}\right), \\
a \otimes \beta & =\left(u_{a} u_{\beta},\left(v_{a}^{q}+v_{\beta}^{q}-v_{a}^{q} v_{\beta}^{q}\right)^{1 / q}\right), \\
\gamma a & =\left(\sqrt[q]{1-\left(1-u_{a}^{q}\right)^{\gamma}}, v_{a}^{\gamma}\right), \\
a^{\gamma} & =\left(u_{a}^{\gamma}, \sqrt[q]{1-\left(1-v_{a}^{q}\right)^{\gamma}}\right) .
\end{aligned}
$$


Definition 3 (see [33]). Let $\widehat{a}=\left(a, b, c, d ; u_{\widehat{a}}, v_{\widehat{a}}\right)$ be a PTrFN, and its membership function and nonmembership function, respectively, satisfy equations (4) and (5):

$$
\begin{aligned}
& u_{\hat{a}}(x)= \begin{cases}\frac{(x-a) u_{\hat{a}}}{b-a}, & \text { if }(a \leq x<b), \\
u_{\hat{a}}, & \text { if }(b \leq x \leq c), \\
\frac{(x-d) u_{\hat{a}}}{d-c}, & \text { if }(c<x \leq d), \\
0, & \text { if }(x<a, x>d),\end{cases} \\
& v_{\widehat{a}}(x)= \begin{cases}\frac{\left[b-x+v_{\hat{a}}(x-a)\right]}{b-a}, & \text { if }(a \leq x<b), \\
v_{\widehat{a}}, & \text { if }(b \leq x \leq c), \\
\frac{\left[x-c+v_{\tilde{a}}(d-x)\right]}{d-c}, & \text { if }(c<x \leq d), \\
1, & \text { if }(x<a, x>d),\end{cases}
\end{aligned}
$$

where $u_{\hat{a}}$ is the maximum degree of membership of $\widehat{a}$ and $v_{\hat{a}}$ is the minimum degree of nonmembership of $\hat{a}$, which satisfy $0 \leq u_{\hat{a}} \leq 1,0 \leq v_{\hat{a}} \leq 1$, and $0 \leq\left(u_{\hat{a}}\right)^{2}+\left(v_{\widehat{a}}\right)^{2} \leq 1$.

Definition 4 (see [33]). Let $\widehat{a}=\left(a_{\widehat{a}}, b_{\widehat{a}}, c_{\widehat{a}}, d_{\widehat{a}} ; u_{\widehat{a}}, v_{\widehat{a}}\right)$ and $\widehat{b}=$ $\left(a_{\hat{b}}, b_{\hat{b}}, c_{\hat{b}}, d_{\hat{b}} ; u_{\hat{b}}, v_{\hat{b}}\right)$ be two PTrFNs; $\gamma>0$ is random number; then, the following equations hold:

$$
\begin{aligned}
& \widehat{a} \oplus \widehat{b}=\left(a_{\widehat{a}}+a_{\widehat{b}}, b_{\widehat{a}}+b_{\widehat{b}}, c_{\widehat{a}}+c_{\widehat{b}}, d_{\widehat{a}}+d_{\widehat{b}} ; \sqrt{u_{\widehat{a}}^{2}+u_{\hat{b}}^{2}-u_{\hat{a}}^{2} u_{\hat{b}}^{2}}, v_{\widehat{a}} v_{\widehat{b}}\right), \\
& \widehat{a} \otimes \widehat{b}=\left(a_{\widehat{a}} a_{\widehat{b}}, b_{\widehat{a}} b_{\widehat{b}}, c_{\widehat{a}} c_{\widehat{b}}, d_{\widehat{a}} d_{\widehat{b}} ; u_{\hat{a}} u_{\hat{b}}, \sqrt{v_{\widehat{a}}^{2}+v_{\widehat{b}}^{2}-v_{\widehat{a}}^{2} v_{\widehat{b}}^{2}}\right), \\
& \gamma \widehat{a}=\left(\gamma a_{\widehat{a}}, \gamma b_{\widehat{a}}, \gamma c_{\widehat{a}}, \gamma d_{\widehat{a}} ; \sqrt{1-\left(1-u_{\widehat{a}}^{2}\right)^{\gamma}}, v_{\widehat{a}}^{\gamma}\right), \\
& \hat{a}^{\gamma}=\left(d_{a}^{\gamma}, c_{a}^{\gamma}, b_{a}^{\gamma}, a_{a}^{\gamma} ; u_{a}^{\gamma}, \sqrt{1-\left(1-v_{a}^{2}\right)^{\gamma}}\right) .
\end{aligned}
$$

\section{3. $q$-Rung Orthopair Trapezoidal Fuzzy Numbers}

This section first puts forward the definition of $q$-ROTrFN and investigates the operation low of $q$-ROTrFNs. Then, a new ranking method of $q$-ROTrFNs, a $q$-ROTrFWA operator, and $q$-rung orthopair trapezoidal fuzzy Hamming distance measure are developed, respectively.

Definition 5. Let $\widetilde{a}=\left(\underline{a}, a_{1}, a_{2}, \bar{a} ; u_{\tilde{a}(\max )}, v_{\tilde{a}(\min )}\right)$ be a $q$ ROTrFN; its membership function $u_{\tilde{a}}$ is shown as

$$
u_{\tilde{a}}(x)= \begin{cases}\frac{(x-\underline{a}) u_{\mathfrak{a}(\max )},}{a_{1}-\underline{a}}, & \text { if }\left(\underline{a} \leq x<a_{1}\right), \\ u_{\tilde{a}(\max )} & \text { if }\left(a_{1} \leq x \leq a_{2}\right), \\ \frac{(\bar{a}-x) u_{\tilde{a}(\max )},}{\bar{a}-a_{2}} & \text { if }\left(a_{2}<x \leq \bar{a}\right), \\ 0, & \text { if }(x<\underline{a}, x>\bar{a}) .\end{cases}
$$

And, the nonmembership function $v_{a}^{\text {is shown as }}$ 


$$
v_{\tilde{a}}(x)= \begin{cases}\frac{\left[a_{1}-x+v_{\tilde{a}(\min )}(x-\underline{a})\right]}{a_{1}-\underline{a}}, & \text { if }\left(\underline{a} \leq x<a_{1}\right), \\ v_{\tilde{a}(\min )}, & \text { if }\left(a_{1} \leq x \leq a_{2}\right), \\ \frac{\left[x-a_{2}+v_{a(\min )}(\bar{a}-x)\right]}{\bar{a}-a_{2}}, & \text { if }\left(a_{2}<x \leq \bar{a}\right), \\ 1, & \text { if }(x<\underline{a}, x>\bar{a}),\end{cases}
$$

where $0 \leq u_{\tilde{a}(\max )} \leq 1, \quad 0 \leq v_{\mathfrak{a}(\min )} \leq 1$, and $0 \leq\left(u_{\tilde{a}(\max )}\right)^{q}+$ $\left(v_{a(\min )}\right)^{q} \leq 1, q \geq 1$.

As shown in Figures 1 and 2, the $q-\operatorname{ROTrFN} \widetilde{a}$ is different from the PTrFN and the intuitionistic trapezoidal fuzzy number (ITrFN), and obviously, the $q$-ROTrFNs are more flexible because of $0 \leq\left(u_{\tilde{a}(\max )}\right)^{q}+\left(v_{\tilde{a}(\min )}\right)^{q} \leq 1$.

$\pi_{\tilde{a}}(x)$ is the degree of indeterminacy of the $q-\operatorname{ROTrFN} \tilde{a}$ and is shown as

$$
\pi_{\tilde{a}}(x)=\sqrt[q]{1-u_{a}^{q}(x)-v_{a}^{q}(x)} .
$$

It is easy to see that trapezoidal fuzzy numbers, ITrFN and PTrFN, are the special cases of the developed $q$-ROTrFN. That is to say, the $q$-ROTrFN degenerates to an ITrFN when

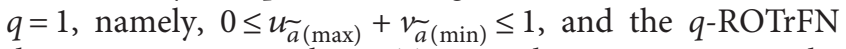
degenerates into the PTrFN when $q=2$, namely, $0 \leq\left(u_{\tilde{a}(\max )}\right)^{2}+\left(v_{\tilde{a}(\min )}\right)^{2} \leq 1$.

Definition 6. Let $\gamma$ be a random number and $\gamma>0$ and $\tilde{a}=$ $\left(\underline{a}, a_{1}, a_{2}, \bar{a} ; u_{\tilde{a}(\max )}, v_{a(\min )}\right) \quad$ and $\quad \tilde{b}=\left(\underline{b}, b_{1}, b_{2}, \bar{b} ; u_{b(\max )}\right.$, $\left.v_{b(\min )}\right)$ be two $q$-ROTrFNs; then, the following equations (13)-(16) hold:

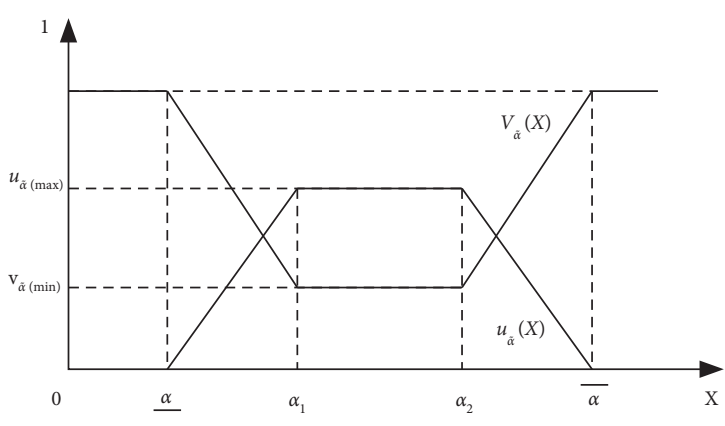

FIgURE 1: $q$-ROTrFN $\tilde{a}=\left(\underline{a}, a_{1}, a_{2}, \bar{a} ; \mathfrak{w}_{\tilde{a}(\max )}, v_{a(\min )}\right)$.

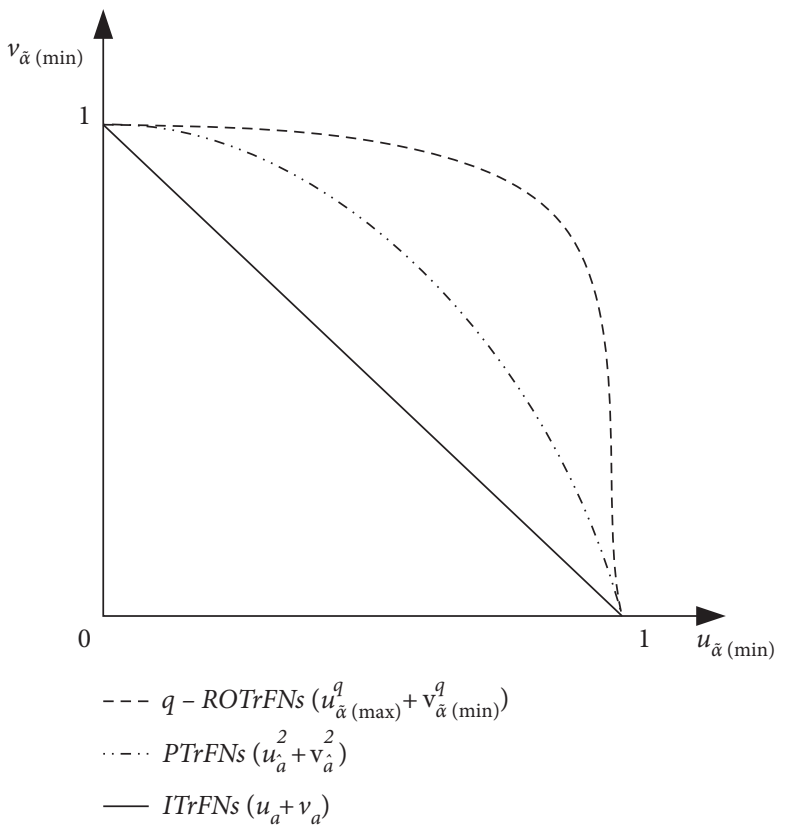

FigURE 2: Information space with different values of $q$.

$$
\begin{aligned}
& \tilde{a} \oplus \widetilde{b}=\left(\underline{a}+\underline{b}, a_{1}+b_{1}, a_{2}+b_{2}, \bar{a}+\bar{b} ; \sqrt[q]{u_{a(\max )}^{q}+u_{b(\max )}^{q}-u_{a(\max )}^{q} u_{b(\max )}^{q}}, v_{a(\min )} v_{b(\min )}\right), \\
& \tilde{a} \otimes \widetilde{b}=\left(\underline{a} \underline{b}, a_{1} b_{1}, a_{2} b_{2}, \bar{a} \bar{b} ; u_{\tilde{a}(\max )} u_{\tilde{b}(\max )}, \sqrt[q]{v_{\tilde{a}(\min )}^{q}+v_{\tilde{b}(\min )}^{q}-v_{\tilde{a}(\min )}^{q} \frac{v^{q}(\min )}{q}}\right), \\
& \gamma \widetilde{a}=\left(\gamma \underline{\underline{a}}, \gamma a_{1}, \gamma a_{2}, \gamma \bar{a} ; \sqrt[q]{1-\left(1-u_{a}^{q}(\max )\right)^{\gamma}}, v_{\widetilde{a}(\min )}^{\gamma}\right), \\
& \widetilde{a}^{\gamma}=\left(\bar{a}^{\gamma}, a_{2}^{\gamma}, a_{1}^{\gamma}, \underline{\underline{a}} ; u_{a(\max )}^{\gamma}, \sqrt[q]{1-\left(1-v_{\tilde{a}(\min )}^{q}\right)^{\gamma}}\right) .
\end{aligned}
$$

Theorem 1. Given two q-ROTrFNs $\tilde{a}=\left(\underline{a}, a_{1}, a_{2}, \bar{a} ; u_{\tilde{a}(\max )}\right.$, $\left.v_{a(\min )}\right)$ and $\widetilde{b}=\left(\underline{b}, b_{1}, b_{2}, \bar{b} ; u_{b(\max )}, v_{b(\min )}\right)$, and $\gamma>0, \lambda>0$; then, we have

(1) $\tilde{a} \oplus \widetilde{b}=\widetilde{b} \oplus \widetilde{a}$

(2) $\widetilde{a} \otimes \widetilde{b}=\widetilde{b} \otimes \widetilde{a}$
(3) $\gamma(\widetilde{a} \oplus \widetilde{b})=\gamma \widetilde{a} \oplus \gamma \tilde{b}$

(4) $\gamma \widetilde{a} \oplus \lambda \widetilde{a}=(\gamma+\lambda) \widetilde{a}$ 
Proof

(1)

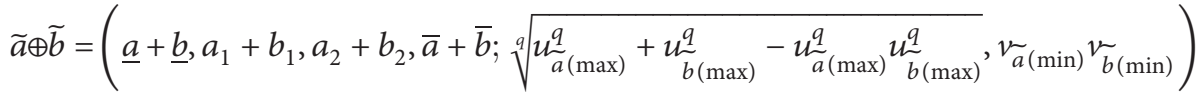

$$
\begin{aligned}
& =\left(\underline{b}+\underline{a}, b_{1}+a_{1}, b_{2}+a_{2}, \bar{b}+\bar{a} ; \sqrt[q]{u_{b(\max )}^{q}+u_{a(\max )}^{q}-u_{b(\max )}^{q} u_{a(\max )}^{q}}, v_{b(\min )} v_{a(\min )}\right) \\
& =\widetilde{b} \oplus \widetilde{a} \text {. }
\end{aligned}
$$

(2) The same as (1) can be proved, $\tilde{a} \otimes \widetilde{b}=\widetilde{b} \otimes \widetilde{a}$.

(3)

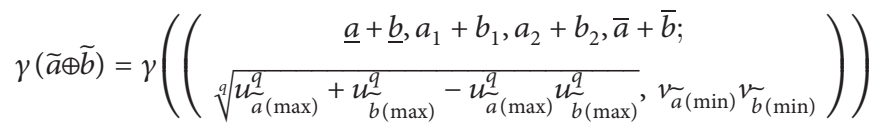

$$
\begin{aligned}
& =\left(\gamma(\underline{a}+\underline{b}), \gamma\left(a_{1}+b_{1}\right), \gamma\left(a_{2}+b_{2}\right), \gamma(\bar{a}+\bar{b}) ; \sqrt[q]{1-\left[\left(1-u_{a(\max )}^{q}\right)\left(1-u_{b(\max )}^{q}\right)\right]^{\gamma}},\left(v_{a(\min )} v_{b(\min )}\right)^{\gamma}\right)
\end{aligned}
$$

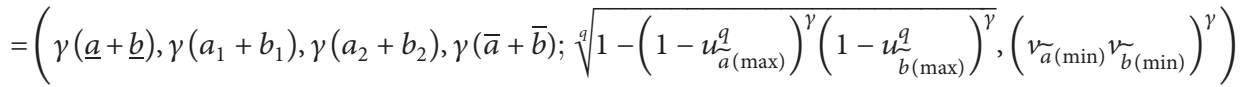

$$
\begin{aligned}
& =\left(\gamma(\underline{a}+\underline{b}), \gamma\left(a_{1}+b_{1}\right), \gamma\left(a_{2}+b_{2}\right), \gamma(\bar{a}+\bar{b}) ;\right. \\
& {\left[\begin{array}{c}
1-\left(1-u_{a(\max )}^{q}\right)^{\gamma}+1-\left(1-u_{b(\max )}^{q}\right)^{\gamma}- \\
\left.\left.q\left[1-\left(1-u_{b(\max )}^{q}\right)^{\gamma}-\left(1-u_{a(\max )}^{q}\right)^{\gamma}+\left(1-u_{a(\max )}^{q}\right)^{\gamma}\left(1-u_{b(\max )}^{q}\right)^{\gamma}\right]^{,\left(\nu_{a}(\min )\right.} v_{b(\min )}\right)^{\gamma}\right)
\end{array}\right.} \\
& =\left(\gamma(\underline{a}+\underline{b}), \gamma\left(a_{1}+b_{1}\right), \gamma\left(a_{2}+b_{2}\right), \gamma(\bar{a}+\bar{b}) ; \sqrt[q]{\begin{array}{l}
1-\left(1-u_{a(\max )}^{q}\right)^{\gamma}+1-\left(1-u_{b(\max )}^{q}\right)^{\gamma}- \\
{\left[1-\left(1-u_{b(\max )}^{q}\right)^{\gamma}\right]\left[1-\left(1-u_{b(\max )}^{q}\right)^{\gamma}\right]^{\prime}}
\end{array},\left(v_{\tilde{a}(\min )}^{\left.v_{b(\min )}\right)^{\gamma}}\right.}\right) \\
& =\gamma \widetilde{a} \oplus \gamma \tilde{b} .
\end{aligned}
$$

(4) $\quad \gamma \widetilde{a}=\left(\gamma \underline{a}, \gamma a_{1}, \gamma a_{2}, \gamma \bar{a} ; \sqrt[q]{1-\left(1-u_{a(\max )}^{q}\right)^{\gamma}}, v_{\tilde{a}(\min )}^{\gamma}\right)$

$$
\begin{aligned}
& \lambda \widetilde{a}=\left(\lambda \underline{a}, \lambda a_{1}, \lambda a_{2}, \lambda \bar{a} ; \sqrt[q]{1-\left(1-u_{a(\max )}^{q}\right)^{\lambda}}, v_{a(\min )}^{\lambda}\right) \\
& \gamma \tilde{a} \oplus \lambda \tilde{a}=\left(\begin{array}{l}
(\gamma+\lambda) \underline{a},(\gamma+\lambda) a_{1},(\gamma+\lambda) a_{2},(\gamma+\lambda) \bar{a} ; \sqrt[q]{1-\left(1-u_{a(\max )}^{q}\right)^{\gamma}+1-\left(1-u_{\tilde{a}(\max )}^{q}\right)^{\lambda}-} \sqrt{\left[1-\left(1-u_{a(\max )}^{q}\right)^{\gamma}\right]\left[1-\left(1-u_{a}^{q}(\max )\right)^{\lambda}\right.}, v_{\tilde{a}(\min )}^{\gamma} \nu_{a(\min )}^{\lambda}
\end{array}\right. \\
& \left.=\left((\gamma+\lambda) \underline{a},(\gamma+\lambda) a_{1},(\gamma+\lambda) a_{2},(\gamma+\lambda) \bar{a} ; \sqrt[q]{1-\left(1-u_{a}^{q}(\max )\right.}\right)^{\gamma}\left(1-u_{a(\max )}^{q}\right)^{\lambda}, v_{\widetilde{a}(\min )}^{\gamma} v_{\tilde{a}(\min )}^{\lambda}\right) \\
& \left.=\left((\gamma+\lambda) \underline{a},(\gamma+\lambda) a_{1},(\gamma+\lambda) a_{2},(\gamma+\lambda) \bar{a} ; \sqrt[q]{1-\left(1-u_{a}^{q}(\max )\right.}\right)^{\gamma+\lambda}, v_{\tilde{a}(\min )}^{\gamma+\lambda}\right) \\
& =(\gamma+\lambda) \widetilde{a} .
\end{aligned}
$$


Example 1. Let $\tilde{a}=(2,3,5,7 ; 0.7,0.5) \quad$ and $\widetilde{b}=(1,4,6,8 ; 0.8,0.3)$ be two $q$-ROTrFNs; $\gamma=2, \lambda=3$, and $q=3$; then, we have

$$
\begin{aligned}
& \tilde{a} \oplus \widetilde{b}=\left(2+1,3+4,5+6,7+8 ; \sqrt[3]{0.7^{3}+0.8^{3}-0.7^{3} \times 0.8^{3}}, 0.5 \times 0.3\right) \\
& =(3,7,11,15 ; 0.8791,0.15)
\end{aligned}
$$

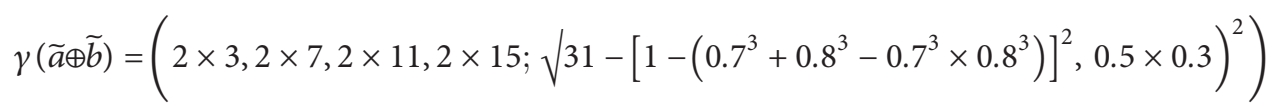

$$
\begin{aligned}
& =(6,14,22,30 ; 0.9645,0.0225)
\end{aligned}
$$

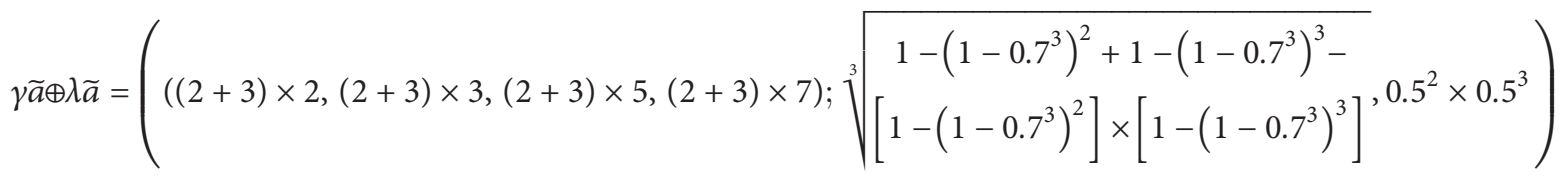

$$
\begin{aligned}
& =(10,15,25,35 ; 0.9574,0.03125) \text {. }
\end{aligned}
$$

Definition 7. Let $\widetilde{a}=\left(\underline{a}, a_{1}, a_{2}, \bar{a} ; u_{\tilde{a}(\max )}, v_{\tilde{a}(\min )}\right)$ be a $q$ ROTrFN, $q \geq 1$; then, the score function of $\widetilde{a}$ is shown as

$$
S(\widetilde{a})=\left(\frac{\underline{a}+a_{1}+a_{2}+\bar{a}}{4}\right) \times\left(u \frac{q}{a(\max )}-v \frac{q}{a(\min )}\right) .
$$

Definition 8. Let $\tilde{a}=\left(\underline{a}, a_{1}, a_{2}, \bar{a} ; u_{\tilde{a}(\max )}, v_{\tilde{a}(\min )}\right)$ be a $q$ ROTrFN, $q \geq 1$; then, the exact function of $\widetilde{a}$ is shown as

$$
H(\widetilde{a})=\left(\frac{\underline{a}+a_{1}+a_{2}+\bar{a}}{4}\right) \times\left(u_{\tilde{a}(\max )}^{q}+v_{\tilde{a}(\min )}^{q}\right) .
$$

Obviously, $H(\widetilde{a})$ reflects the degree of accuracy of $\widetilde{a}$, and $\tilde{a}$ has become more accurate as $H(\widetilde{a})$ increases. Then, the comparison method of $q$-ROTrFNs according to Definitions 7 and 8 can be determined.

Definition 9. Let $\widetilde{a}=\left(\underline{a}, a_{1}, a_{2}, \bar{a} ; w_{\tilde{a}(\max )}, v_{a(\min )}\right)$ and $\tilde{b}=$ $\left(\underline{b}, b_{1}, b_{2}, \bar{b} ; u_{b(\max )}, v_{b(\min )}\right)$ be two $q$-ROTrFNs; $q \geq 1, S(\widetilde{a})$, $S(\widetilde{b}), H(\widetilde{a})$, and $H(b)$ are the score function and exact function of $\widetilde{a}$ and $\widetilde{b}$, respectively; then, the ranking method for $q$-ROTrFNs is introduced as follows:

(1) If $S(\tilde{a})>S(\tilde{b})$, then $\tilde{a}>\tilde{b}$

(2) If $S(\tilde{a})<S(\tilde{b})$, then $\tilde{a}<\tilde{b}$

(3) When $S(\widetilde{a})=S(\widetilde{b})$, if $H(\widetilde{a})>H(\tilde{b})$, then $\widetilde{a}>\widetilde{b}$

$$
\begin{aligned}
& \text { If } H(\widetilde{a})<H(\widetilde{b}) \text {, then } \tilde{a}<\tilde{b} \\
& \text { If } H(\widetilde{a})=H(\tilde{b}) \text {, then } \widetilde{a}=\widetilde{b}
\end{aligned}
$$

Example 2. Given two $q$-ROTrFNs $\tilde{a}=(1,5,6,7 ; 0.8,0.5)$ and $\vec{b}=(4,7,8,9 ; 0.3,0.9)$ and $q=2$, then we use the developed ranking method to compare the magnitudes of these two $q$-ROTrFNs as below:

$$
\begin{aligned}
& S(\widetilde{a})=\left(\frac{1+5+6+7}{4}\right) \times(0.64-0.25)=1.8525 \\
& S(\tilde{b})=\left(\frac{4+7+8+9}{4}\right) \times(0.09-0.81)=-5.04
\end{aligned}
$$

Obviously, $S(\tilde{b})<S(\widetilde{a})$, so it can be found that $\tilde{a}>\widetilde{b}$.

However, if $\tilde{a}=(4,5,5,6 ; 0.4,0.3) \quad$ and $\widetilde{b}=(5,7,7,9 ; 0.3,0.2), \quad q=2$, it can be obtained that $S(\widetilde{a})=S(\widetilde{b})=0.35$. Then, the relationship between $\tilde{a}$ and $\widetilde{b}$ has to be determined by an exact function:

$$
\begin{aligned}
& H(\widetilde{a})=\left(\frac{4+5+5+6}{4}\right) \times(0.16+0.09)=1.25 \\
& H(\tilde{b})=\left(\frac{5+7+7+9}{4}\right) \times(0.09+0.04)=0.91 .
\end{aligned}
$$

Obviously, $H(\widetilde{b})<H(\widetilde{a})$, which means $\widetilde{a}>\widetilde{b}$.

On the contrary, if $\tilde{a}=(1,5,6,7 ; 0.8,0.5)$ and $\widetilde{b}=(4,7,8,9 ; 0.3,0.9), q=3$, we have

$$
\begin{aligned}
& S(\widetilde{a})=\left(\frac{1+5+6+7}{4}\right) \times(0.512-0.125)=1.83825, \\
& S(\tilde{b})=\left(\frac{4+7+8+9}{4}\right) \times(0.027-0.729)=-4.914
\end{aligned}
$$

It is easy to find $\widetilde{a}>\widetilde{b}$. Similarly, if $\widetilde{a}=(4,5,5,6 ; 0.4,0.3)$ and $\widetilde{b}=(5,7,7,9 ; 0.3,0.2), q=3$, it can be obtained that $S(\widetilde{a})=0.185>S(\widetilde{b})=0.133$. Therefore, $\widetilde{a}>\widetilde{b}$.

Next, we propose a new $q$-ROTrFWA operator and investigate its properties.

Definition 10. Let $\tilde{a}=\left(\tilde{a}_{1}, \tilde{a}_{2}, \ldots, \tilde{a}_{n}\right)^{T}$ be a set of $q$ ROTrFNs and $\omega=\left(\omega_{1}, \omega_{2}, \ldots, \omega_{n}\right)^{T}$ be the weight vector of $\tilde{a}_{i}(i=1,2, \ldots, n), \omega_{i} \in[0,1]$ and $\sum_{i=1}^{n} \omega_{i}=1$; then, the definition of $q$-ROTrFWA is shown as 


$$
q-\operatorname{ROTrFWA}\left(\widetilde{a}_{1}, \widetilde{a}_{2}, \ldots, \widetilde{a}_{n}\right)=\sum_{i=1}^{n} \omega_{i} \widetilde{a}_{i}=\omega_{1} \widetilde{a}_{1} \oplus \omega_{2} \widetilde{a}_{2} \oplus \cdots \oplus \omega_{n} \widetilde{a}_{n} .
$$

Theorem 2. The aggregating result obtained by $q-\operatorname{ROTrFWA}\left(\widetilde{a}_{1}, \widetilde{a}_{2}, \ldots, \widetilde{a}_{n}\right)$ is still a q-ROTrFN, which is shown as

$$
q-\operatorname{ROTrFWA}\left(\tilde{a}_{1}, \tilde{a}_{2}, \ldots, \tilde{a}_{n}\right)=\left(\sum_{i=1}^{n} \omega_{i} \tilde{a}_{i}^{\underline{a}} \sum_{i=1}^{n} \omega_{i} \tilde{a}_{i}^{a_{1}}, \sum_{i=1}^{n} \omega_{i} \tilde{a}_{i}^{a_{2}}, \sum_{i=1}^{n} \omega_{i} \tilde{a}_{i}^{\bar{a}} ; \sqrt{\left(1-\prod_{i=1}^{n}\left(1-u_{a_{i}(\max )}^{q}\right)^{\omega_{i}}\right)}, \prod_{i=1}^{n} v_{\tilde{a}_{i}(\min )}^{\omega_{i}}\right) .
$$

Proof. Theorem 2 can be proved by mathematical induction as follows:

(1) Obviously, when $n=1$, the equation holds.

$$
q-\operatorname{ROTrFWA}\left(\tilde{a}_{1}, \tilde{a}_{2}, \ldots, \widetilde{a}_{m}\right)=\left(\sum_{i=1}^{m} \omega_{i} \tilde{a}_{i}^{a} \sum_{i=1}^{m} \omega_{i} \tilde{a}_{i}^{a_{1}}, \sum_{i=1}^{m} \omega_{i} \widetilde{a}_{i}^{a_{2}}, \sum_{i=1}^{m} \omega_{i} \tilde{a}_{i}^{\bar{a}} ; \sqrt[p]{\left(1-\prod_{i=1}^{m}\left(1-u_{a_{i}(\max )}^{q}\right)^{\omega_{i}}\right)}, \prod_{i=1}^{m} v_{\widetilde{a}_{i}(\min )}^{\omega_{i}}\right) .
$$

When $n=m+1$,

$$
\omega_{m+1} \widetilde{a}_{m+1}=\left(\omega_{m+1} \tilde{a}_{m+1}^{\underline{a}}, \omega_{m+1} \tilde{a}_{m+1}^{a_{1}}, \omega_{m+1} \tilde{a}_{m+1}^{a_{2}}, \omega_{m+1} \tilde{a}_{m+1}^{\bar{a}} ; \sqrt[q]{\left(1-\left(1-u{\frac{q}{a_{m+1}(\max )}}^{q}\right)\right.}, v_{m+1(\min )}^{\omega_{m+1}}\right) .
$$

According to equation (8), the result is as follows:

$$
\begin{aligned}
& q-\operatorname{ROTrFWA}\left(\tilde{a}_{1}, \tilde{a}_{2}, \ldots, \tilde{a}_{m}\right) \oplus \omega_{m+1} \tilde{a}_{m+1} \\
& =\left(\sum_{i=1}^{m} \omega_{i} \tilde{a}_{i}^{\underline{a}+\omega_{m+1}} \tilde{a} 1_{i}^{\underline{a}} \sum_{i=1}^{m} \omega_{i} \tilde{a}\right. \\
& \left.\sqrt{q\left(\begin{array}{c}
\left(1-\prod_{i=1}^{m}\left(1-u_{\tilde{a}_{i}}(\max )^{q}\right)^{\omega_{i}}\right)+\left(1-\left(1-u_{\tilde{a}_{m+1}}(\max )^{q}\right)^{\omega_{m+1}}\right) \\
\left(1-\prod_{i=1}^{m}\left(1-u_{\tilde{a}_{i}}(\max )^{q}\right)^{\omega_{i}}\right) \times\left(1-\left(1-u_{\tilde{a}_{m+1}}(\max )^{q}\right)^{\omega_{m+1}}\right)
\end{array}\right),\left(\prod_{i=1}^{m}\left(v_{a} i(\min )\right)^{\omega_{i}}\right) \times v_{m+1(\min )}^{\omega_{m+1}}}\right) \\
& =\left(\sum_{i=1}^{m+1} \omega_{i} \underline{a}_{i} \tilde{a}_{i=1}^{m+1} \omega_{i} \tilde{a} i^{a_{1}}, \sum_{i=1}^{m+1} \omega_{i} \widetilde{a}_{i}^{a_{2}}, \sum_{i=1}^{m+1} \omega_{i} \widetilde{a}_{i}^{\bar{a}} ; \sqrt[p]{\left(1-\prod_{i=1}^{m+1}\left(1-u_{\tilde{a}_{m+1}}(\max )^{q}\right)^{\omega_{i}}\right)}, \prod_{i=1}^{m+1}\left(v_{a_{i}}(\min )\right)^{\omega_{i}}\right)
\end{aligned}
$$

$=q-\operatorname{ROTrFWA}\left(\widetilde{a}_{1}, \tilde{a}_{2}, \ldots, \tilde{a}_{m+1}\right)$. 
It can be seen from the proof that, for any integer $n$, the collective result obtained by the $q$-ROTrFWA operator is still a $q$-ROTrFN.

Example 3. Given four $q$-ROTrFNs $\widetilde{a}=(0.2,0.3,0.5,0.6$; $0.6,0.3), \quad \widetilde{b}=(0.3,0.5,0.6,0.7 ; 0.3,0.8), \quad \widetilde{c}=(0.2,0.4,0.5$,
$0.6 ; 0.8,0.5)$, and $\widetilde{d}=(0.1,0.5,0.7,0.8 ; 0.7,0.5)$, and $q=2$ and the corresponding weights of four $q$-ROTrFNs $\omega_{a}=0.15, \omega_{b}=0.35, \omega_{c}=0.2$, and $\omega_{d}^{\sim}=0.3$, then the result obtained by the $q$-ROTrFWA is shown as follows:

$$
\begin{aligned}
& q-\operatorname{ROTrFWA}(\widetilde{a}, \widetilde{b}, \widetilde{c}, \widetilde{d}) \\
& =\omega_{a} \widetilde{a} \oplus \omega_{b} \tilde{b} \oplus \omega_{c} \widetilde{c} \oplus \omega_{d} \widetilde{d} \\
& =(0.15 \times(0.2,0.3,0.5,0.6 ; 0.6,0.3)) \oplus(0.35 \times(0.3,0.5,0.6,0.7 ; 0.3,0.8)) \\
& \oplus(0.2 \times(0.2,0.4,0.5,0.6 ; 0.8,0.5)) \oplus(0.3 \times(0.1,0.5,0.7,0.8 ; 0.7,0.5)) \\
& =(0.03,0.05,0.08,0.09 ; 0.25,0.83) \oplus(0.11,0.18,0.21,0.25 ; 0.18,0.92) \\
& \oplus(0.02,0.1,0.14,0.16 ; 0.35,0.87) \oplus(0.03,0.15,0.21,0.24 ; 0.43,0.81) \\
& =(0.19,0.48,0.64,0.74 ; 0.59,0.54) .
\end{aligned}
$$

Definition 11. Let $\widetilde{a}=\left(\underline{a}, a_{1}, a_{2}, \bar{a} ; u_{\tilde{a}(\max }, v_{\tilde{a}(\min )}\right)$ and $\widetilde{b}=$ $\left(\underline{b}, b_{1}, b_{2}, \bar{b} ; u_{\tilde{b}(\max )}, v_{b(\min )}\right)$ be two $q$-ROTrFNs; the Hamming distance $d(\tilde{a}, \tilde{b})$ between $\tilde{a}$ and $\tilde{b}$ is show as

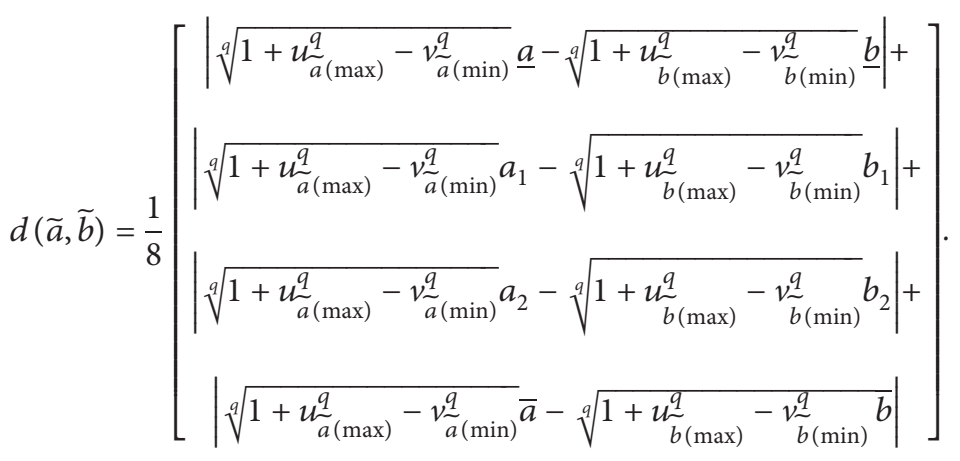

Example 4. Given two $q$-ROTrFNs $\widetilde{a}=(0.4,0.7,0.8,1$; $0.6,0.5)$ and $\widetilde{b}=(0.5,0.7,1,2 ; 0.6,0.7)$ and $q=3$, we get the Hamming distance between them as follows:

$$
d(\widetilde{a}, \tilde{b})=\frac{1}{8}\left[\begin{array}{c}
\left|\sqrt[3]{1+0.6^{3}-0.5^{3}} \times 0.4-\sqrt[3]{1+0.6^{3}-0.7^{3}} \times 0.5\right|+ \\
\left|\sqrt[3]{1+0.6^{3}-0.5^{3}} \times 0.7-\sqrt[3]{1+0.6^{3}-0.7^{3}} \times 0.7\right|+ \\
\left|\sqrt[3]{1+0.6^{3}-0.5^{3}} \times 0.8-\sqrt[3]{1+0.6^{3}-0.7^{3}} \times 1\right|+
\end{array}\right]=0.141
$$

As a classical distance measure, the developed Hamming distance provides a way to quantify the difference between $q$ ROTrFNs, which indicates the closeness of two $q$-ROTrFNs and takes into account the multifactor differences of $q$ ROTrFNs. 


\section{A Modified TODIM Group Decision-Making Method for $q$-ROTrFNs}

In this section, we develop a new $q$-rung orthopair trapezoidal fuzzy modified TODIM group decision-making method for solving MAGDM problems with $q$-ROTrFNs. We here consider a common MAGDM problem with a set of feasible alternatives $A=\left\{A_{1}, A_{2}, \ldots, A_{m}\right\}$; each alternative is evaluated based on $n$ attributes $C=\left\{C_{1}, C_{2}, \ldots, C_{n}\right\}$, where the weight of attribute $C_{j}$ can be expressed as $\omega_{j}$, and fulfills $0 \leq \omega_{j} \leq 1$ and $\sum_{j=1}^{n} \omega_{j}=1$. Let $P=\left\{P_{1}, P_{2}, \ldots, P_{t}\right\}$ be the set of experts; the weight of the expert $P_{k}$ can be expressed as $\lambda_{k}$ and the weights of different experts satisfy $\sum_{k=1}^{t} \lambda_{k}=1, \lambda_{k} \geq 0(k=1,2, \ldots, t)$. For each expert, the decision matrix can be obtained according to different alternatives and attributes. For the $k$ th expert, the decision matrix is expressed as $A^{(k)}=\left(\widetilde{a}_{i j}^{(k)}\right)_{m \times n}, k=1,2, \ldots, t$. The element $\tilde{a}_{i j}^{(k)}$ in the decision matrix means that the evaluated data of the alternative $A_{\mathrm{i}}$ on the attribute $C_{j}$ is provided by the expert $P_{k}$, and each $\tilde{a}_{i j}^{(k)}$ is a q-ROTrFN. On the basis of the developed $q$-ROTrFWA operator, we can integrate the individual decision matrices $A^{(k)}=\left(\widetilde{a}_{i j}^{(k)}\right)_{m \times n}(k=1,2, \ldots, t)$ into the collective decision matrix $R=\left(\widetilde{r}_{i j}\right)_{m \times n}$ as follows:

$$
\tilde{r}_{i j}=q-\operatorname{ROTrFWA}\left(\tilde{a}_{i j}^{(1)}, \widetilde{a}_{i j}^{(2)}, \ldots, \widetilde{a}_{i j}^{(t)}\right), \quad i=1,2, \ldots, m, j=1,2, \ldots, n .
$$

After obtaining the collective decision matrix $R=\left(\widetilde{r}_{i j}\right)_{m \times n}$, we next measure the relative dominance of each alternative over the others by constructing the prospect value function [25]. Firstly, the attribute with the highest weight can be regarded as the reference attribute according to the TODIM method $[21,25]$ and then the relative weight $\omega_{\text {cr }}$ of the attribute $C_{c}$ to the reference attribute $C_{r}$ can be obtained as

$$
\omega_{\mathrm{cr}}=\frac{\omega_{c}}{\omega_{r}}
$$

where $\omega_{r}=\max \left\{\omega_{c} \mid c=1,2, \ldots, n\right\}$ is the reference weight.

To calculate the relative dominance degree of each alternative, this section first proposes a new $q$-rung orthopair trapezoidal fuzzy distance measure-based compromise approach. The compromise method can take fully into account the importance of positive-ideal alternative and negative-ideal alternative in decision-making and well avoid the defect of Hamming distance that sometimes cannot reflect the distinction of $q$-ROTrFNs.

Definition 12. If $A=\left(\widetilde{a}_{i j}\right)_{m \times n}=\left(\left(\underline{a}, a_{1}, a_{2}, \bar{a} ; \mathfrak{u}_{\tilde{a}(\max )}\right.\right.$, $\left.\left.v_{a(\min )}\right)_{i j}\right)_{m \times n}$ is a $q$-ROTrFNs matrix given by the expert, then the positive-ideal set $A^{+}$and the negative-ideal set $A^{-}$ of the alternatives are shown as

$$
A^{+}=\left(\widetilde{a}_{1}^{+}, \widetilde{a}_{2}^{+}, \ldots, \widetilde{a}_{m}^{+}\right),
$$

where

$$
\begin{aligned}
& \widetilde{a}_{i}^{+}=\left(\left(\max _{1 \leq j \leq n}\left\{\underline{a}_{i j}\right\}, \max _{1 \leq j \leq n}\left\{a_{1_{i j}}\right\}, \max _{1 \leq j \leq n}\left\{a_{2_{i j}}\right\}, \max _{1 \leq j \leq n}\left\{\bar{a}_{i j}\right\} ; \max _{1 \leq j \leq n}\left\{u_{a_{i j}(\max )}\right\}, \min _{1 \leq j \leq n}\left\{v_{a_{i j}(\min )}\right\}\right)\right), \\
& A^{-}=\left(\widetilde{a}_{1}^{-}, \tilde{a}_{2}^{-}, \ldots, \widetilde{a}_{m}^{-}\right),
\end{aligned}
$$

where

$$
\tilde{a}_{i}^{-}=\left(\left(\min _{1 \leq j \leq n}\left\{\underline{a}_{i j}\right\}, \min _{1 \leq j \leq n}\left\{a_{1_{i j}}\right\}, \min _{1 \leq j \leq n}\left\{a_{2_{i j}}\right\}, \min _{1 \leq j \leq n}\left\{\bar{a}_{i j}\right\} ; \min _{1 \leq j \leq n}\left\{u_{a_{i j}(\max )}\right\}, \max _{1 \leq j \leq n}\left\{v_{a_{i j}(\min )}\right\}\right)\right) .
$$

Definition 13. The distance $D^{+}$between each element $a_{i j}$ in the $q$-ROTrFNs matrix and the positive-ideal alternative $A^{+}=\left(\tilde{a}_{1}^{+}, \tilde{a}_{2}^{+}, \ldots, \tilde{a}_{m}^{+}\right)$, and the distance $D^{-}$between each element $a_{i j}$ and the negative-ideal alternative $A^{-}=\left(\widetilde{a}_{1}^{-}, \widetilde{a}_{2}^{-}, \ldots, \widetilde{a}_{m}^{-}\right)$are defined as equations (39) and (40), respectively: 


$$
\begin{aligned}
& D^{+}\left(\tilde{a}_{i j}\right)=\omega_{j} d\left(\widetilde{a}_{i j}, \tilde{a}_{i}^{+}\right) \\
& =\frac{1}{8} \omega_{j}\left[\left|\sqrt[q]{1+u_{a_{i j}(\max )}^{q}-v{\frac{a}{a_{i j}(\min )}}_{a_{i j}}^{q}}-\sqrt[q]{1+u_{a_{i}^{+}(\max )}^{q}-v_{\tilde{a}_{i}^{+}(\min )}^{q}} \underline{a}_{i}^{+}\right|\right. \\
& +\left|\sqrt[q]{1+u{\frac{a_{i j}(\max )}{q}}^{q} v_{a_{i j}(\min )}^{q}} a_{1_{i j}}-\sqrt[q]{1+u_{a_{i}^{+}(\max )}^{q}-v_{\widetilde{a}_{i}^{+}(\min )}^{q}} a_{1_{i}^{+}}^{+}\right| \\
& +\left|\sqrt[q]{1+u_{a_{i j}(\max )}^{q}-v_{\tilde{a}_{i j}(\min )}^{q}} a_{2_{i j}}-\sqrt[q]{1+u_{\tilde{a}_{i}^{+}(\max )}^{q}-v_{\tilde{a}_{i}^{+}(\min )}^{q}} a_{2_{i}^{+}}^{+}\right| \\
& \left.+\left|\sqrt[q]{1+u_{a_{i j}(\max )}^{q}-v_{a_{i j}(\min )}^{q}} \bar{a}_{i j}-\sqrt[q]{1+u_{a_{i}(\max )}^{q}-v_{\widetilde{a}_{i}^{+}(\min )}^{q}} \bar{a}_{i}^{+}\right|\right], \\
& D^{-}\left(\widetilde{a}_{i j}\right)=\omega_{j} d\left(\widetilde{a}_{i j}, \widetilde{a}_{i}^{-}\right) \\
& =\frac{1}{8} \omega_{j}\left[\left|\sqrt[q]{1+u_{a_{i j}(\max )}^{q}-v v_{a_{i j}(\min )}^{q}} \frac{a_{i j}}{}-\sqrt[q]{1+u{\frac{a_{i}}{(\max )}}^{q}-v v_{a_{i}}^{q}(\min )} \underline{a}_{i}^{-}\right|\right. \\
& +\left|\sqrt[q]{1+u{\frac{a}{a_{i j}(\max )}}^{q}-v{\frac{a}{a_{i j}(\min )}}_{1_{i j}}^{q}}-\sqrt[q]{1+u \frac{q}{a_{i}(\max )}-v{\frac{a_{i}}{(\min )}}_{1_{i_{i}}}^{q}}\right| \\
& +\left|\sqrt[q]{1+u \tilde{a}_{i j}^{q}(\max )}-v_{\tilde{a}_{i j}(\min )}^{q} a_{2_{i j}}-\sqrt[q]{1+u_{a_{i}}^{q}(\max )}-v_{\widetilde{a}_{i}(\min )}^{q} a_{2_{i}}^{-}\right|
\end{aligned}
$$

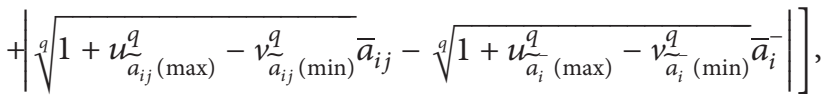

where $\omega_{j}$ is the relative weight of the attribute, $0 \leq \omega_{j} \leq 1$ and $\sum_{j=1}^{m} \omega_{j}=1$. Obviously, $D^{+}$and $D^{-}$are the further expansion of $q$-ROTrFNs based on Hamming distance, which can fully consider the impacts of benefit and cost on decisionmaking. However, how to deal with MADM via $D^{+}$and $D^{-}$ is still the focus of the follow-up research.

In order to find a compromise, a method to express the relative importance of $D^{+}$and $D^{-}$by the parameters $\alpha$ and $(1-\alpha)$ is as follows, where $0 \leq \alpha \leq 1$. Based on the proposed distance measure function, the distance-based compromise with $q$-ROTrFNs is defined as follows.

Definition

14. Let $A=\left(\tilde{a}_{i j}\right)_{m \times n}=\left(\left(\underline{a}, a_{1}, a_{2}, \bar{a} ; u_{\tilde{a}(\max )}, v_{a(\min )}\right)_{i j}\right)_{m \times n}$ be a $q-$ rung orthopair trapezoidal fuzzy evaluation matrix; the compromise value of the element $a_{i j}$ is shown as

$$
D\left(\widetilde{a}_{i j}\right)=\alpha D^{-}\left(\widetilde{a}_{i j}\right)-(1-\alpha) D^{+}\left(\widetilde{a}_{i j}\right) .
$$

It is easy to find that $0 \leq D\left(\widetilde{a}_{i j}\right) \leq 1$. By setting an appropriate distance parameter $\alpha$, the compromise value of $\widetilde{a}_{i}$ can be obtained according to its positive-ideal distance $D^{+}$ and negative-ideal distance $D^{-}$. On this basis, a distance measure-based compromise approach is proposed to distinguish $q$-ROTrFNs.

Definition 15. Let $\tilde{a}=\left(\underline{a}, a_{1}, a_{2}, \bar{a} ; u_{a(\max )}, v_{\tilde{a}(\min )}\right)$ and $\widetilde{b}=$ $\left(\underline{b}, b_{1}, b_{2}, \bar{b} ; u_{b(\max )}, v_{b(\min )}\right)$ be two $q$-ROTrFNs; then, the distance measure-based compromise value $\operatorname{Cd}(\widetilde{a}, \widetilde{b})$ between $\tilde{a}$ and $\tilde{b}$ is shown as

$$
\operatorname{Cd}(\widetilde{a}, \widetilde{b})=|D(\widetilde{a})-D(\widetilde{b})|,
$$

Compared with the Hamming distance proposed in Definition 11, the compromise distance is more accurate and comprehensive when applied to decision-making methods. Based on the Hamming distance, the compromise distance can fully consider the importance of the two aspects of the 
ideal alternatives in the decision-making and is more feasible in actual decision-making. Next, we use Example 5 to show the advantage of the compromise distance.
Example 5. Consider a $q$-rung orthopair trapezoidal fuzzy matrix $R$ as

$$
R=\left\{\begin{array}{c}
(0.52,0.40,0.40,0.34 ; 0.71,0.46),(0.45,0.42,0.55,0.47 ; 0.57,0.53), \\
(0.38,0.33,0.43,0.44 ; 0.71,0.46),(0.44,0.4,0.42,0.40 ; 0.48,0.66) \\
(0.49,0.34,0.39,0.43 ; 0.70,0.46),(0.35,0.34,0.40,0.37 ; 0.41,0.68), \\
(0.40,0.53,0.64,0.50 ; 0.51,0.58),(0.38,0.44,0.3,0.29 ; 0.57,0.57) \\
(0.42,0.38,0.41,0.44 ; 0.62,0.51),(0.39,0.41,0.36,0.48 ; 0.49,0.58), \\
(0.41,0.45,0.28,0.32 ; 0.29,0.67),(0.55,0.74,0.74,0.43 ; 0.50,0.70) \\
(0.57,0.67,0.46,0.22 ; 0.67,0.58),(0.52,0.34,0.30,0.38 ; 0.37,0.75), \\
(0.37,0.42,0.5,0.46 ; 0.61,0.6),(0.37,0.47,0.45,0.52 ; 0.52,0.65)
\end{array}\right\} .
$$

According to equations (36) and (38), the positive-ideal set $A^{+}$and the negative-ideal set $A^{-}$of the alternatives can be obtained as

$$
\begin{aligned}
& A^{+} \quad=\left(\widetilde{a}_{1}^{+}, \tilde{a}_{2}^{+}, \tilde{a}_{3}^{+}, \tilde{a}_{4}^{+}\right)= \\
&\left(\begin{array}{l}
(0.52,0.42,0.55,0.47 ; 0.71,0.46),(0.49,0.53,0.64,0.50 ; 0.70,0.46), \\
(0.55,0.74,0.74,0.48 ; 0.62,0.51),(0.57,0.67,0.50,0.52 ; 0.67,0.58)
\end{array}\right), \\
& A^{-} \quad=\left(\widetilde{a}_{1}^{-}, \widetilde{a}_{2}^{-}, \widetilde{a}_{3}^{-}, \widetilde{a}_{4}^{-}\right)= \\
& \quad\left(\begin{array}{l}
(0.38,0.33,0.40,0.34 ; 0.48,0.66),(0.35,0.34,0.30,0.29 ; 0.41,0.68), \\
(0.39,0.38,0.28,0.32 ; 0.29,0.70),(0.37,0.34,0.30,0.22 ; 0.37,0.71)
\end{array}\right) .
\end{aligned}
$$

Let $\alpha=0.5$ and $q=2$; the Hamming distance and the compromise distance for $R$ are shown in the Figure 3.

Obviously, the Hamming distance is greater than the compromise distance according to Figure 3. The reason for the situation is that the compromise distance takes into account the difference between the positive- and negativeideal distances of alternatives compared to the Hamming distance. In the light of this characteristic of the compromise distance, it will be more comprehensive when dealing with the deviation between $q$-ROTrFNs, and it can also be seen from the figure that the compromise distance is more stable. Therefore, this paper will further apply the compromise distance to modify the TODIM method for group decisionmaking.
By using the above developed compromise distance, we can calculate the relative dominance degree of the alternative $A_{i}$ over the alternative $A_{j}$ concerning the attribute $C_{c}$ using the following expression:

$$
\Phi_{c}\left(A_{i}, A_{j}\right)= \begin{cases}\sqrt{\frac{\omega_{c r}}{\sum_{c=1}^{n} \omega_{c r}} \operatorname{Cd}\left(\tilde{r}_{i c}, \widetilde{r}_{j c}\right)}, & \text { if }\left(\tilde{r}_{i c}>\widetilde{r}_{j c}\right), \\ 0, & \text { if }\left(\tilde{r}_{i c}=\widetilde{r}_{j c}\right), \\ -\frac{1}{\theta} \sqrt{\frac{\sum_{c=1}^{n} \omega_{c r}}{\omega_{c r}} \operatorname{Cd}\left(\tilde{r}_{i c}, \tilde{r}_{j c}\right)}, & \text { if }\left(\tilde{r}_{i c}<\tilde{r}_{j c}\right),\end{cases}
$$


Input: $q$-ROTrFNs matrices

Output: ranking result according to the value of $\xi_{i}$

Process

Step 1: to eliminate the influence of different physical dimensions on decision-making, transform the evaluation matrix into a standard matrix $A^{(k)}, k=1,2, \ldots, t$.

Step 2: decision maker chooses the value of $\theta$ according to the risk preference and gets the value of $q$ by the observation method or the traversal method. The value of $q$ needs to hold $0 \leq\left(u_{a_{(k)}(\max )}\right)^{q}+\left(v_{\sim_{(k)}(\min }\right)^{q} \leq 1$ in matrix $A^{(k)}, k=1,2, \ldots, t$.

Step 3: aggregate $A^{(k)}$ by equation (34), $\tilde{a}_{i j}^{(t)}$ represents $q$-ROTrFNs in the decision matrix given by the $t$ th expert, and get the collective matrix $R=\left(\widetilde{r}_{i j}\right)_{m \times n}$.

Step 4: get the relative weight of each attribute $C_{c}$ using equation (35).

Step 5: obtain the positive-ideal alternative $A^{+}=\left(\tilde{a}_{1}^{+}, \tilde{a}_{2}^{+}, \ldots, \tilde{a}_{m}^{+}\right)$and the negative-ideal alternative $A^{-}=\left(\tilde{a}_{1}^{-}, \tilde{a}_{2}^{-}, \ldots, \tilde{a}_{m}^{-}\right)$of $A=$ $\left\{A_{1}, A_{2}, \ldots, A_{m}\right\}$ according to equations (36) and (38) and using equations (39) and (40) to get the distance between them and each element in $R=\left(\widetilde{r}_{i j}\right)_{m \times n}$.

Step 6: the compromise distances of elements in $\tilde{r}_{i j}$ are obtained via equations (41) and (42).

Step 7: get the dominance of alternative $A_{i}$ relative to alternative $A_{j}$ by equations (45) and (46).

Step 8: calculate the overall dominance $\xi_{i}$ by equation (47).

Step 9: according to the value of $\xi_{i}$, the ranking result of the alternatives is the same as rank of $\xi_{i}$, and the alternative with the largest $\xi_{i}$ is the optimal.

Algorithm 1: Algorithm of the modified TODIM method.

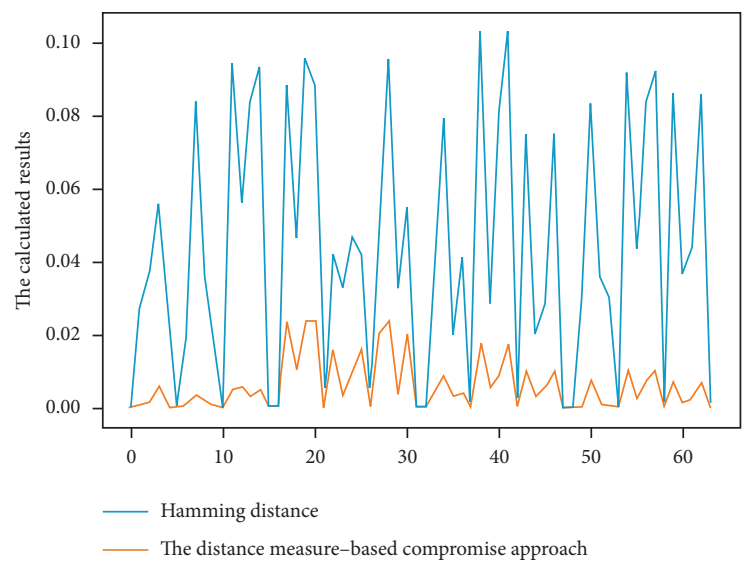

FIgURE 3: Difference between Hamming distance and compromise distance.

where $\tilde{r}_{i c}$ represents a $q$-ROTrFN in the matrix and $\mathrm{Cd}\left(\widetilde{r}_{i c}, \widetilde{r}_{j c}\right)$ reflects the compromise distance between two $q-$ ROTrFNs. $\theta(\theta>0)$ is the attenuation factor, and the smaller the value of the parameter $\theta$, the higher loss avoidance of the expert.

Obviously, there exist three cases in equation (45). (1) If $\widetilde{r}_{i c}>\widetilde{r}_{j c}$, then $\Phi_{c}\left(A_{i}, A_{j}\right)$ represents a gain. (2) If $\widetilde{r}_{i c}=\widetilde{r}_{j c}$, then $\Phi_{c}\left(A_{i}, A_{j}\right)$ represents a nil. (3) If $\widetilde{r}_{i c}<\widetilde{r}_{j c}$, then $\Phi_{c}\left(A_{i}, A_{j}\right)$ represents a loss.
By aggregating $\phi_{c}\left(A_{i}, A_{j}\right)$ with each attribute $C_{c}$, the dominance of the alternative $A_{i}$ over the alternative $A_{j}$ can be obtained as follows:

$$
\vartheta\left(A_{i}, A_{j}\right)=\sum_{c=1}^{n} \phi_{c}\left(A_{i}, A_{j}\right), \quad i, j=1,2, \ldots, m .
$$

Finally, we calculate the overall dominance degree of the alternative $A_{i}$ according to the following expression:

$$
\xi\left(A_{i}\right)=\frac{\sum_{k=1}^{m} \vartheta\left(A_{i}, A_{k}\right)-\min _{i}\left\{\sum_{k=1}^{m} \vartheta\left(A_{i}, A_{k}\right)\right\}}{\max _{i}\left\{\sum_{k=1}^{m} \vartheta\left(A_{i}, A_{k}\right)\right\}-\min _{i}\left\{\sum_{k=1}^{m} \vartheta\left(A_{i}, A_{k}\right)\right\}}, \quad i=1,2, \ldots, m .
$$

Obviously, $0 \leq \xi\left(A_{i}\right) \leq 1$, and the greater $\xi\left(A_{i}\right)$ is, the better the alternative $A_{i}$ will be. Therefore, the ranking order of all alternatives $A_{i}(i=1,2, \ldots, m)$ can be determined according to the increasing order of the dominance degree of the alternative $A_{i}(i=1,2, \ldots, m)$. The algorithm of the $q$-rung orthopair trapezoidal fuzzy modified TODIM group decision-making method is introduced in Algorithm 1. 
The process of the group decision-making method is shown in Figure 4.

\section{Case Study on Health Management of Hypertensive Patients}

In this section, a case study on health management of hypertensive patients is conducted to demonstrate the feasibility of the modified TODIM group decision-making method, and the comparison analysis with the existing methods and sensitive analysis of different parameters are investigated in detail.

5.1. Decision Results Obtained by the Developed Modified TODIM Method. At present, there are about 245 million hypertensive patients in China, and related diseases caused by hypertension have brought a heavy burden to residents and society. In order to improve the health of residents, the health management of hypertensive patients has been highly valued by the public and the government. In order to simplify the management process, a community hospital plans to develop hypertension health management software to facilitate the treatment and management of patients by community doctors, which means that doctors need to evaluate the risk level based on the residents' inspection. The main observation data include blood pressure measurement, cardiovascular damage, related diseases (mainly related to cardiovascular and cerebrovascular diseases), and related risk factors (age, smoking, drinking and sleeping, eating more sodium and less potassium, greasy, obesity, work and life pressure, etc.), and the weights of them are $\omega=(0.4,0.3,0.2,0.1)$. In order to better provide prompts to doctors, the risk level of residents is indicated by different colors: red indicates high risk, orange indicates moderate risk, blue indicates mild danger, and green indicates normal, which are expressed as $A=\left\{A_{1}, A_{2}, A_{3}, A_{4}\right\}$.

Considering that these four indicators are abnormal to the normal process, the process is ascending, adjusting, and gradually recovering. The fuzzy decision model is used to construct the system for processing. In order to facilitate the flexible evaluation of experts, the $q$-ROTrFNs are used for evaluation. Now, a 65-year-old resident has a systolic blood pressure of $140 \mathrm{mmHg}$ and a diastolic blood pressure of $99 \mathrm{mmHg}$. He suffers from hyperglycemia, and parents suffer from high blood pressure. Besides, he smokes and drinks frequently, and his taste tends to be heavy. Four experts are invited to evaluate the risk level of this resident, and the weights of experts are $\lambda=(0.3,0.3,0.2,0.2)$. The evaluation data are obtained in Tables 1-4.

Now, we employ the developed modified q-rung orthopair trapezoidal fuzzy TODIM group decision-making method to help the doctor identify the risk level of hypertensive patients. In this case, we consider $q=2, \theta=1$, and $\alpha=0.5$; then, we aggregate the evaluation matrix $A^{(k)}(k=$ $1,2,3,4)$ given by these four experts to obtain the collective matrix $R=\left(\widetilde{r}_{i j}\right)_{m \times n}$ by equation (34), as shown in Table 5 .
According to the definition of compromise distance, we can calculate the compromise distance between two risk levels under each attribute as below:

$$
\begin{aligned}
& \operatorname{Cd}\left(\widetilde{r}_{11} \widetilde{r}_{21}\right)=8.69 \times 10^{-3}, \\
& \operatorname{Cd}\left(\widetilde{r}_{11} \widetilde{r}_{31}\right)=3.14 \times 10^{-2} \text {, } \\
& \operatorname{Cd}\left(\widetilde{r}_{11} \widetilde{r}_{41}\right)=1.67 \times 10^{-2} \text {, } \\
& \operatorname{Cd}\left(\widetilde{r}_{21} \widetilde{r}_{31}\right)=4.01 \times 10^{-2} \text {, } \\
& \operatorname{Cd}\left(\widetilde{r}_{21} \widetilde{r}_{41}\right)=2.54 \times 10^{-2} \text {, } \\
& \operatorname{Cd}\left(\widetilde{r}_{31} \widetilde{r}_{41}\right)=1.47 \times 10^{-2} \text {, } \\
& \operatorname{Cd}\left(\widetilde{r}_{12} \widetilde{r}_{22}\right)=4.87 \times 10^{-2} \text {, } \\
& \operatorname{Cd}\left(\widetilde{r}_{12} \widetilde{r}_{32}\right)=1.41 \times 10^{-2} \text {, } \\
& \mathrm{Cd}\left(\widetilde{r}_{12} \widetilde{r}_{42}\right)=3.11 \times 10^{-2} \text {, } \\
& \mathrm{Cd}\left(\widetilde{r}_{22} \widetilde{r}_{32}\right)=3.46 \times 10^{-2} \text {, } \\
& \mathrm{Cd}\left(\widetilde{r}_{22} \widetilde{r}_{42}\right)=1.76 \times 10^{-2} \text {, } \\
& \operatorname{Cd}\left(\widetilde{r}_{32} \widetilde{r}_{42}\right)=1.70 \times 10^{-2} \text {, } \\
& \mathrm{Cd}\left(\widetilde{r}_{13} \widetilde{r}_{23}\right)=1.42 \times 10^{-2} \text {, } \\
& \operatorname{Cd}\left(\widetilde{r}_{13} \widetilde{r}_{33}\right)=8.97 \times 10^{-3} \text {, } \\
& \mathrm{Cd}\left(\widetilde{r}_{13} \widetilde{r}_{43}\right)=2.01 \times 10^{-2} \text {, } \\
& \mathrm{Cd}\left(\widetilde{r}_{23} v_{33}\right)=5.21 \times 10^{-3} \text {, } \\
& \operatorname{Cd}\left(\widetilde{r}_{23} \widetilde{r}_{43}\right)=5.97 \times 10^{-3} \text {, } \\
& \mathrm{Cd}\left(\widetilde{r}_{33} \widetilde{r}_{43}\right)=1.12 \times 10^{-2} \text {, } \\
& \mathrm{Cd}\left(\widetilde{r}_{14} \widetilde{r}_{24}\right)=7.95 \times 10^{-3} \text {, } \\
& \operatorname{Cd}\left(\widetilde{r}_{14} \widetilde{r}_{34}\right)=2.75 \times 10^{-3} \text {, } \\
& \mathrm{Cd}\left(\widetilde{r}_{14} \widetilde{r}_{44}\right)=3.90 \times 10^{-4} \text {, } \\
& \mathrm{Cd}\left(\widetilde{r}_{24} \widetilde{r}_{34}\right)=5.19 \times 10^{-3} \text {, } \\
& \mathrm{Cd}\left(\widetilde{r}_{24} \widetilde{r}_{44}\right)=8.34 \times 10^{-3} \text {, } \\
& \operatorname{Cd}\left(\widetilde{r}_{34} \widetilde{r}_{44}\right)=3.14 \times 10^{-3} \text {. }
\end{aligned}
$$

Then, the relative weight of the attribute $C_{c}(c=1,2,3,4)$ can be determined by equation (35), and the result is as follows:

$$
\omega_{\mathrm{cr}}=(1,0.75,0.5,0.25) .
$$

Next, the relative dominance matrix of the risk level $A_{i}$ relative to the risk level $A_{j}$ can be determined by equations (45) and (46), respectively, and the results are shown as below:

$$
\vartheta=\left(\begin{array}{cccc}
0 & -0.19 & 0.28 & 0.40 \\
-0.60 & 0 & 0.27 & 0.36 \\
-1.10 & -0.89 & 0 & 0.33 \\
-1.58 & -1.42 & -1.35 & 0
\end{array}\right) \text {. }
$$




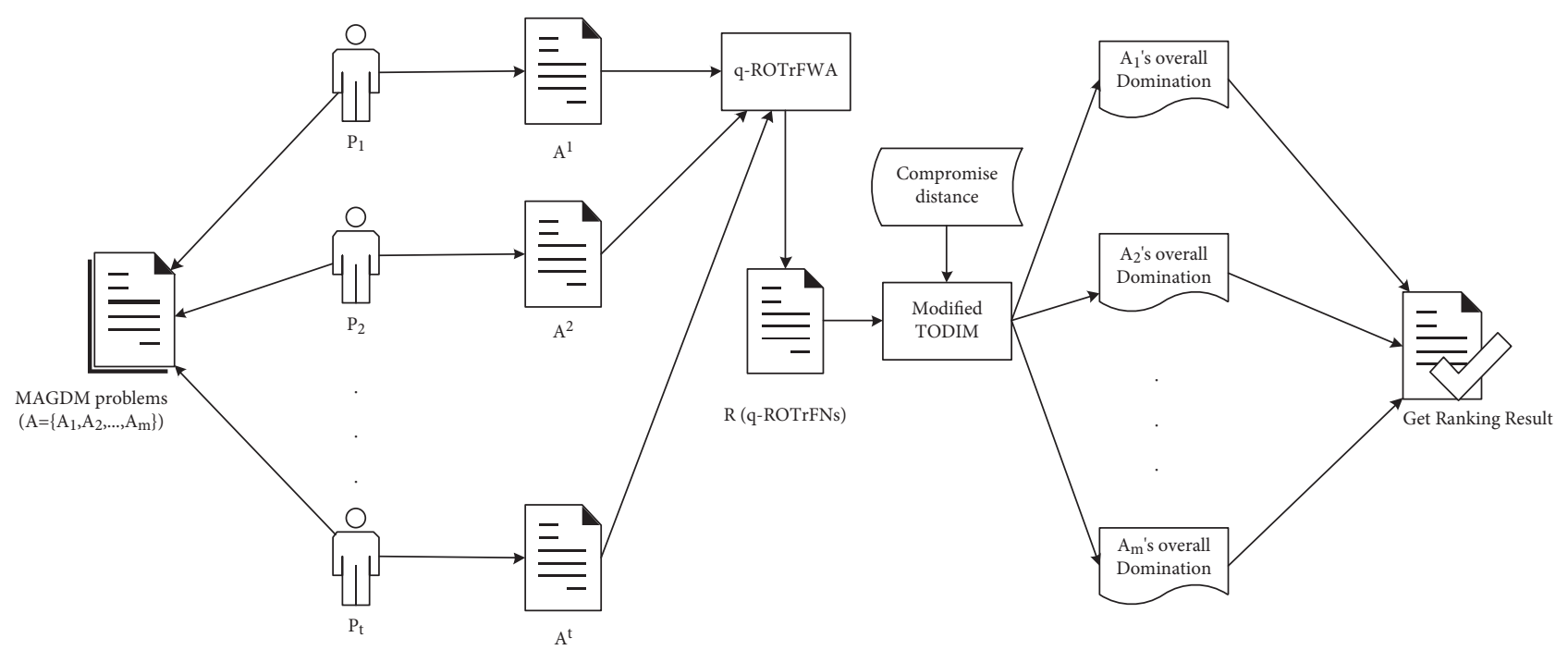

FIgURE 4: The process of the group method with $q$-ROTrFNs.

TABLe 1: Evaluation value matrix by $\operatorname{expert}_{1}\left(A^{1}\right)$.

\begin{tabular}{ccccc}
\hline & $C_{1}$ & $C_{2}$ & $C_{3}$ & $C_{4}$ \\
\hline$A_{1}$ & $(0.3,0.5,0.6,0.8 ; 0.9,0.3)$ & $(0.1,0.2,0.3,0.4 ; 0.6,0.3)$ & $(0.5,0.6,0.8,0.9 ; 0.8,0.1)$ & $(0.4,0.5,0.6,0.7 ; 0.7,0.4)$ \\
$A_{2}$ & $(0.6,0.7,0.8,0.9 ; 0.8,0.5)$ & $(0.5,0.6,0.7,0.8 ; 0.7,0.3)$ & $(0.4,0.5,0.7,0.8 ; 0.9,0.4)$ & $(0.1,0.3,0.5,0.6 ; 0.8,0.3)$ \\
$A_{3}$ & $(0.1,0.2,0.4,0.5 ; 0.7,0.4)$ & $(0.2,0.3,0.5,0.6 ; 0.5,0.4)$ & $(0.5,0.6,0.7,0.8 ; 0.6,0.2)$ & $(0.3,0.4,0.5,0.7 ; 0.6,0.5)$ \\
$A_{4}$ & $(0.3,0.4,0.5,0.6 ; 0.2,0.7)$ & $(0.1,0.2,0.4,0.5 ; 0.4,0.5)$ & $(0.2,0.3,0.5,0.7 ; 0.5,0.5)$ & $(0.1,0.3,0.4,0.5 ; 0.4,0.7)$ \\
\hline
\end{tabular}

TABLE 2: Evaluation value matrix by $\operatorname{expert}_{2}\left(A^{2}\right)$.

\begin{tabular}{ccccc}
\hline & $C_{1}$ & $C_{2}$ & $C_{3}$ & $C_{4}$ \\
\hline$A_{1}$ & $(0.2,0.3,0.5,0.7 ; 0.8,0.3)$ & $(0.1,0.2,0.3,0.4 ; 0.6,0.2)$ & $(0.5,0.6,0.8,0.9 ; 0.9,0.1)$ & $(0.2,0.3,0.5,0.7 ; 0.7,0.4)$ \\
$A_{2}$ & $(0.4,0.5,0.6,0.9 ; 0.6,0.2)$ & $(0.5,0.6,0.7,0.8 ; 0.7,0.4)$ & $(0.4,0.5,0.6,0.8 ; 0.8,0.3)$ & $(0.1,0.2,0.4,0.5 ; 0.6,0.3)$ \\
$A_{3}$ & $(0.1,0.3,0.5,0.7 ; 0.5,0.4)$ & $(0.2,0.3,0.4,0.6 ; 0.4,0.8)$ & $(0.3,0.6,0.7,0.8 ; 0.7,0.2)$ & $(0.3,0.4,0.5,0.8 ; 0.6,0.2)$ \\
$A_{4}$ & $(0.2,0.3,0.5,0.6 ; 0.4,0.7)$ & $(0.2,0.4,0.5,0.6 ; 0.5,0.7)$ & $(0.2,0.3,0.5,0.7 ; 0.5,0.5)$ & $(0.4,0.6,0.7,0.9 ; 0.3,0.2)$ \\
\hline
\end{tabular}

TABLE 3: Evaluation value matrix by $\operatorname{expert}_{3}\left(A^{3}\right)$.

\begin{tabular}{ccccc}
\hline & $C_{1}$ & $C_{2}$ & $C_{3}$ & $C_{4}$ \\
\hline$A_{1}$ & $(0.3,0.4,0.6,0.7 ; 0.8,0.4)$ & $(0.1,0.2,0.3,0.5 ; 0.6,0.2)$ & $(0.4,0.6,0.8,0.9 ; 0.8,0.1)$ & $(0.3,0.5,0.6,0.8 ; 0.7,0.4)$ \\
$A_{2}$ & $(0.2,0.3,0.4,0.5 ; 0.7,0.1)$ & $(0.4,0.5,0.7,0.8 ; 0.5,0.3)$ & $(0.4,0.5,0.6,0.7 ; 0.6,0.1)$ & $(0.2,0.3,0.5,0.7 ; 0.6,0.3)$ \\
$A_{3}$ & $(0.2,0.3,0.5,0.6 ; 0.5,0.3)$ & $(0.3,0.4,0.5,0.6 ; 0.4,0.2)$ & $(0.5,0.7,0.8,0.9 ; 0.5,0.2)$ & $(0.3,0.4,0.5,0.7 ; 0.6,0.2)$ \\
$A_{4}$ & $(0.3,0.4,0.5,0.6 ; 0.2,0.6)$ & $(0.1,0.2,0.4,0.5 ; 0.5,0.4)$ & $(0.3,0.4,0.5,0.7 ; 0.4,0.5)$ & $(0.1,0.2,0.4,0.5 ; 0.4,0.2)$ \\
\hline
\end{tabular}

TABLE 4: Evaluation value matrix by $\operatorname{expert}_{4}\left(A^{4}\right)$.

\begin{tabular}{ccccc}
\hline & $C_{1}$ & $C_{2}$ & $C_{3}$ & $C_{4}$ \\
\hline$A_{1}$ & $(0.6,0.7,0.8,0.9 ; 0.8,0.5)$ & $(0.4,0.6,0.8,0.9 ; 0.6,0.3)$ & $(0.2,0.5,0.8,0.9 ; 0.8,0.3)$ & $(0.5,0.6,0.8,0.9 ; 0.9,0.2)$ \\
$A_{2}$ & $(0.3,0.4,0.5,0.7 ; 0.7,0.2)$ & $(0.2,0.4,0.5,0.6 ; 0.5,0.5)$ & $(0.3,0.4,0.5,0.7 ; 0.7,0.4)$ & $(0.2,0.4,0.5,0.6 ; 0.9,0.2)$ \\
$A_{3}$ & $(0.4,0.5,0.7,0.8 ; 0.5,0.4)$ & $(0.2,0.4,0.5,0.6 ; 0.4,0.5)$ & $(0.1,0.5,0.7,0.8 ; 0.4,0.5)$ & $(0.2,0.3,0.4,0.6 ; 0.6,0.4)$ \\
$A_{4}$ & $(0.2,0.4,0.5,0.7 ; 0.1,0.7)$ & $(0.1,0.3,0.4,0.5 ; 0.3,0.3)$ & $(0.2,0.3,0.4,0.7 ; 0.4,0.6)$ & $(0.1,0.2,0.3,0.5 ; 0.3,0.8)$ \\
\hline
\end{tabular}


TABle 5: Aggregating matrix $R=\left(\widetilde{r}_{i j}\right)_{m \times n}$.

\begin{tabular}{ccccc}
\hline & $C_{1}$ & $C_{2}$ & $C_{3}$ & $C_{4}$ \\
\hline$A_{1}$ & $(0.33,0.46,0.61,0.77 ; 0.84,0.35)$ & $(0.16,0.28,0.40,0.52 ; 0.60,0.24)$ & $(0.42,0.58,0.80,0.90 ; 0.84,0.12)$ & $(0.34,0.46,0.61,0.76 ; 0.76,0.35)$ \\
$A_{2}$ & $(0.40,0.50,0.60,0.78 ; 0.71,0.23)$ & $(0.42,0.54,0.66,0.76 ; 0.64,0.36)$ & $(0.38,0.48,0.61,0.76 ; 0.80,0.28)$ & $(0.14,0.29,0.47,0.59 ; 0.76,0.28)$ \\
$A_{3}$ & $(0.18,0.31,0.51,0.64 ; 0.58,0.38)$ & $(0.22,0.34,0.47,0.60 ; 0.43,0.45)$ & $(0.36,0.60,0.72,0.82 ; 0.59,0.24)$ & $(0.28,0.38,0.48,0.71 ; 0.60,0.30)$ \\
$A_{4}$ & $(0.25,0.37,0.50,0.62 ; 0.27,0.68)$ & $(0.13,0.28,0.43,0.53 ; 0.44,0.48)$ & $(0.22,0.32,0.48,0.70 ; 0.46,0.52)$ & $(0.19,0.35,0.47,0.62 ; 0.35,0.38)$ \\
\hline
\end{tabular}

TABLE 6: The overall dominance values of risk levels with different values of $\alpha$ and $\theta$ in case of $q=2$.

\begin{tabular}{cccccccccc}
\hline$\xi_{i}$ & $\alpha=0.1$ & $\alpha=0.2$ & $\alpha=0.3$ & $\alpha=0.4$ & $\alpha=0.5$ & $\alpha=0.6$ & $\alpha=0.7$ & $\alpha=0.8$ & $\alpha=0.9$ \\
\hline \multirow{4}{*}{$\theta=1$} & {$[1.35,0.58$,} & {$[1.30,0.59$,} & {$[1.26,0.60$,} & {$[1.21,0.63$,} & {$[1.16,0.66$,} & {$[1.11,0.69$,} & {$[1.03,0.75$,} & {$[1.03,0.72$,} & {$[1.02,0.70$,} \\
& -0.35, & -0.35, & -0.33, & -0.36, & -0.39, & -0.41, & -0.44, & -0.47, & -0.50, \\
& $-1.79]$ & $-1.78]$ & $-1.76]$ & $-1.76]$ & $-1.75]$ & $-1.74]$ & $-1.74]$ & $-1.74]$ & $-1.74]$ \\
& {$[1.66,0.78$,} & {$[1.60,0.79$,} & {$[1.54,0.80$,} & {$[1.48,0.84$,} & {$[1.42,0.87$,} & {$[1.35,0.91$,} & {$[1.25,0.98$,} & {$[1.27,0.94$,} & {$[1.26,0.92$,} \\
$\theta=1.5$ & -0.21, & -0.21, & -0.19, & -0.22, & -0.24, & -0.26, & -0.29, & -0.31, & -0.34, \\
& $-1.79]$ & $-1.78]$ & $-1.76]$ & $-1.76]$ & $-1.75]$ & $-1.74]$ & $-1.74]$ & $-1.74]$ & $-1.74]$ \\
& {$[1.89,1.01$,} & {$[1.84,1.02$,} & {$[1.79,1.04$,} & {$[1.74,1.07$,} & {$[1.67,1.10$,} & {$[1.58,1.15$,} & {$[1.46,1.22$,} & {$[1.49,1.18$,} & {$[1.50,1.15$,} \\
$\theta=2.25$ & -0.05, & -0.04, & -0.03, & -0.06, & -0.07, & -0.09, & -0.11, & -0.13, & -0.15, \\
& $-1.79]$ & $-1.78]$ & $-1.76]$ & $-1.76]$ & $-1.75]$ & $-1.74]$ & $-1.74]$ & $-1.74]$ & $-1.74]$ \\
\hline
\end{tabular}

Finally, the overall dominance $\xi_{i}(i=1,2,3,4)$ can be determined by equation (47), which is shown as below:

$$
\begin{aligned}
& \xi_{1}=1.16, \\
& \xi_{2}=0.66 \\
& \xi_{3}=-0.39 \\
& \xi_{4}=-1.75 .
\end{aligned}
$$

According to the overall dominances, $\xi_{1}>\xi_{2}>\xi_{3}>\xi_{4}$, the four risk levels are ranked as $A_{1}>A_{2}>A_{3}>A_{4}$, so blood pressure risk of the patient is high risk, and this hypertensive patient needs to control the blood pressure as soon as possible.

5.2. Sensitivity Analysis of Parameters. This section conducts the sensitive analysis for the ranking orders of risk levels by modifying the values of different parameters. Considering the actual decision-making situation, the values of the parameter $q$ are, respectively, as 2, 3, and 4 by using the traversal method. According to the prospect theory, the values of the parameter $\theta$ in the experiments are, respectively, as $1,1.5$, and 2.25. In addition, the influence of the change of the distance parameter $\alpha$ on the ranking results is also not negligible. According to the different values of $q, \alpha$, and $\theta$, the comprehensive results are shown in Tables 6-8, respectively. The corresponding figures are shown in Figures 5-7.
It can be seen from the above figures that the ranking result does not change with different values of $q, \alpha$, and $\theta$, which are always $A_{1}>A_{2}>A_{3}>A_{4}$. In addition, as the risk parameter $\theta$ increases, the advantages of the risk level $A_{1}$ are not changed in this case. It is worth mentioning that, in this case, the change of $\theta$ does not change the overall dominance of $A_{1}$. From the curve in the figures, it can be seen that the change of $q$ has little effect on the value of the overall dominance of risk levels. Besides, it is proved in practical that the increase of $q$ and $\alpha$ will not change the ranking, although they affect the value of overall dominance. Furthermore, it can be known that the ranking results do not be changed when the values of $\theta$ are $1,1.5$, and 2.25, respectively. From the above analysis, the overall dominance of each risk level will not be changed although they are different trends with the change of $q, \alpha$, and $\theta$; the final results will not be affected in the group decision-making method proposed in this paper. The feasibility and effectiveness of this method are verified.

5.3. Comparison Analysis. To well conduct a comparison analysis with the method proposed in [33], in this case, we set the value of $q$ as 2 and the $q$-ROTrFNs in the above example are transformed into the PTrFNs. Then, the PTFWA operator developed in [33] is introduced as follows:

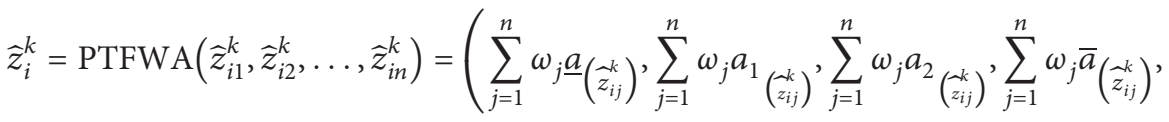

$$
\begin{aligned}
& \left.\left.\sqrt{1-\prod_{j=1}^{n}\left(1-u_{\widehat{a}(\max )}^{2}\left(\begin{array}{c}
-k \\
z_{i j}
\end{array}\right)\right.}\right)^{\omega_{j}}, \prod_{j=1}^{n}\left(v_{\widehat{a}(\min )\left(\bar{z}_{i j}^{k}\right)}^{2}\right)^{\omega_{j}}\right) \text {. }
\end{aligned}
$$


TABle 7: The overall dominance values of risk levels with different values of $\alpha$ and $\theta$ in case of $q=3$.

\begin{tabular}{|c|c|c|c|c|c|c|c|c|c|}
\hline$\xi_{i}$ & $\alpha=0.1$ & $\alpha=0.2$ & $\alpha=0.3$ & $\alpha=0.4$ & $\alpha=0.5$ & $\alpha=0.6$ & $\alpha=0.7$ & $\alpha=0.8$ & $\alpha=0.9$ \\
\hline$\theta=1$ & $\begin{array}{c}{[1.22,0.58} \\
-0.45 \\
-1.82]\end{array}$ & $\begin{array}{c}{[1.19,0.60} \\
-0.46,-1.8]\end{array}$ & $\begin{array}{c}1.15,0.62 \\
-0.47 \\
-1.79]\end{array}$ & $\begin{array}{c}1.11,0.64 \\
-0.48 \\
-1.79]\end{array}$ & $\begin{array}{c}1.06,0.67 \\
-0.50 \\
-1.78]\end{array}$ & $\begin{array}{c}{[1.01,0.70} \\
-0.50 \\
-1.78]\end{array}$ & $\begin{array}{c}0.95,0.76 \\
-0.48 \\
-1.78]\end{array}$ & $\begin{array}{c}0.95,0.71 \\
-0.43 \\
-1.78]\end{array}$ & $\begin{array}{c}{[0.99,0.69} \\
\quad-0.48 \\
-1.78]\end{array}$ \\
\hline$\theta=1.5$ & $\begin{array}{c}{[1.55,0.79} \\
-0.29 \\
-1.82]\end{array}$ & $\begin{array}{c}{[1.49,0.81} \\
-0.30,-1.8]\end{array}$ & $\begin{array}{c}{[1.44,0.83} \\
-0.31 \\
-1.79]\end{array}$ & $\begin{array}{c}{[1.38,0.86} \\
-0.32 \\
-1.79]\end{array}$ & $\begin{array}{c}{[1.32,0.89} \\
-0.34 \\
-1.78]\end{array}$ & $\begin{array}{c}{[1.25,0.93} \\
-0.34 \\
-1.78]\end{array}$ & $\begin{array}{c}{[1.17,0.99} \\
-0.32 \\
-1.78]\end{array}$ & $\begin{array}{c}{[1.18,0.93} \\
-0.29 \\
-1.78]\end{array}$ & $\begin{array}{c}{[1.24,0.91} \\
\quad-0.33 \\
-1.78]\end{array}$ \\
\hline$\theta=2.25$ & $\begin{array}{c}{[1.83,1.04} \\
-0.12 \\
-1.82]\end{array}$ & $\begin{array}{c}{[1.78,1.06} \\
-0.12,-1.8]\end{array}$ & $\begin{array}{c}{[1.73,1.08} \\
-0.13 \\
-1.79]\end{array}$ & $\begin{array}{c}{[1.66,1.11} \\
-0.14 \\
-1.79]\end{array}$ & $\begin{array}{c}{[1.58,1.14} \\
-0.15 \\
-1.78]\end{array}$ & $\begin{array}{c}{[1.49,1.18} \\
-0.16 \\
-1.78]\end{array}$ & $\begin{array}{c}{[1.38,1.24} \\
-0.14 \\
-1.78]\end{array}$ & $\begin{array}{c}{[1.42,1.18} \\
-0.12 \\
-1.78]\end{array}$ & $\begin{array}{c}{[1.50,1.15} \\
\quad-0.16 \\
-1.78]\end{array}$ \\
\hline
\end{tabular}

TABLe 8: The overall dominance values of risk levels with different values of $\alpha$ and $\theta$ in case of $q=4$.

\begin{tabular}{cccccccccc}
\hline$\xi_{i}$ & $\alpha=0.1$ & $\alpha=0.2$ & $\alpha=0.3$ & $\alpha=0.4$ & $\alpha=0.5$ & $\alpha=0.6$ & $\alpha=0.7$ & $\alpha=0.8$ & $\alpha=0.9$ \\
\hline \multirow{3}{*}{$\theta$} & {$[1.16,0.57$,} & {$[1.13,0.59$,} & {$[1.09,0.62$,} & {$[1.05,0.64$,} & {$[1.00,0.67$,} & {$[0.95,0.71$,} & {$[0.90,0.74$,} & {$[0.94,0.71$,} & {$[0.96,0.69$,} \\
& -0.50, & -0.50, & -0.48, & -0.47, & -0.45, & -0.43, & -0.39, & -0.41, & -0.43, \\
& $-1.82]$ & $-1.82]$ & $-1.82]$ & $-1.82]$ & $-1.82]$ & $-1.82]$ & $-1.82]$ & $-1.83]$ & $-1.83]$ \\
& {$[1.48,0.79$,} & {$[1.43,0.81$,} & {$[1.38,0.84$,} & {$[1.32,0.86$,} & {$[1.26,0.90$,} & {$[1.19,0.95$,} & {$[1.13,0.97$,} & {$[1.19,0.93$,} & {$[1.23,0.91$,} \\
$\theta=1.5$ & -0.34, & -0.33, & -0.33, & -0.32, & -0.30, & -0.29, & -0.25, & -0.28, & -0.30, \\
& $-1.82]$ & $-1.82]$ & $-1.82]$ & $-1.82]$ & $-1.82]$ & $-1.82]$ & $-1.82]$ & $-1.83]$ & $-1.83]$ \\
& {$[1.79,1.05$,} & {$[1.75,1.07$,} & {$[1.68,1.09$,} & {$[1.61,1.12$,} & {$[1.53,1.16$,} & {$[1.43,1.21$,} & {$[1.36,1.23$,} & {$[1.45,1.19$,} & {$[1.52,1.17$,} \\
$\theta=2.25$ & -0.15, & -0.15, & -0.14, & -0.14, & -0.13, & -0.12, & -0.09, & -0.12, & -0.14, \\
& $-1.82]$ & $-1.82]$ & $-1.82]$ & $-1.82]$ & $-1.82]$ & $-1.82]$ & $-1.82]$ & $-1.83]$ & $-1.83]$ \\
\hline
\end{tabular}

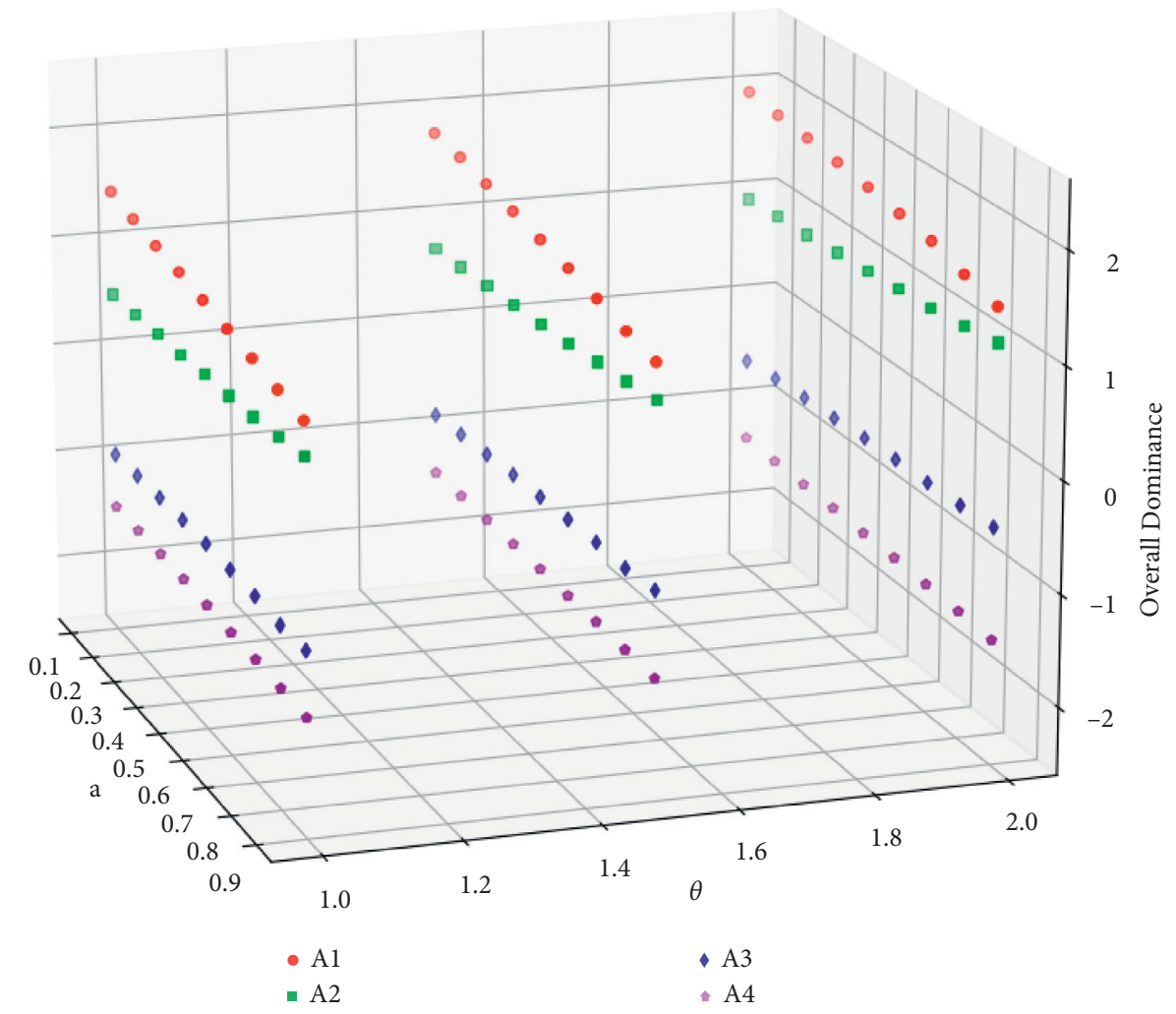

Figure 5: The pictorial expressions of the dominance values with different values of $\alpha$ and $\theta$ in case of $q=2$. 


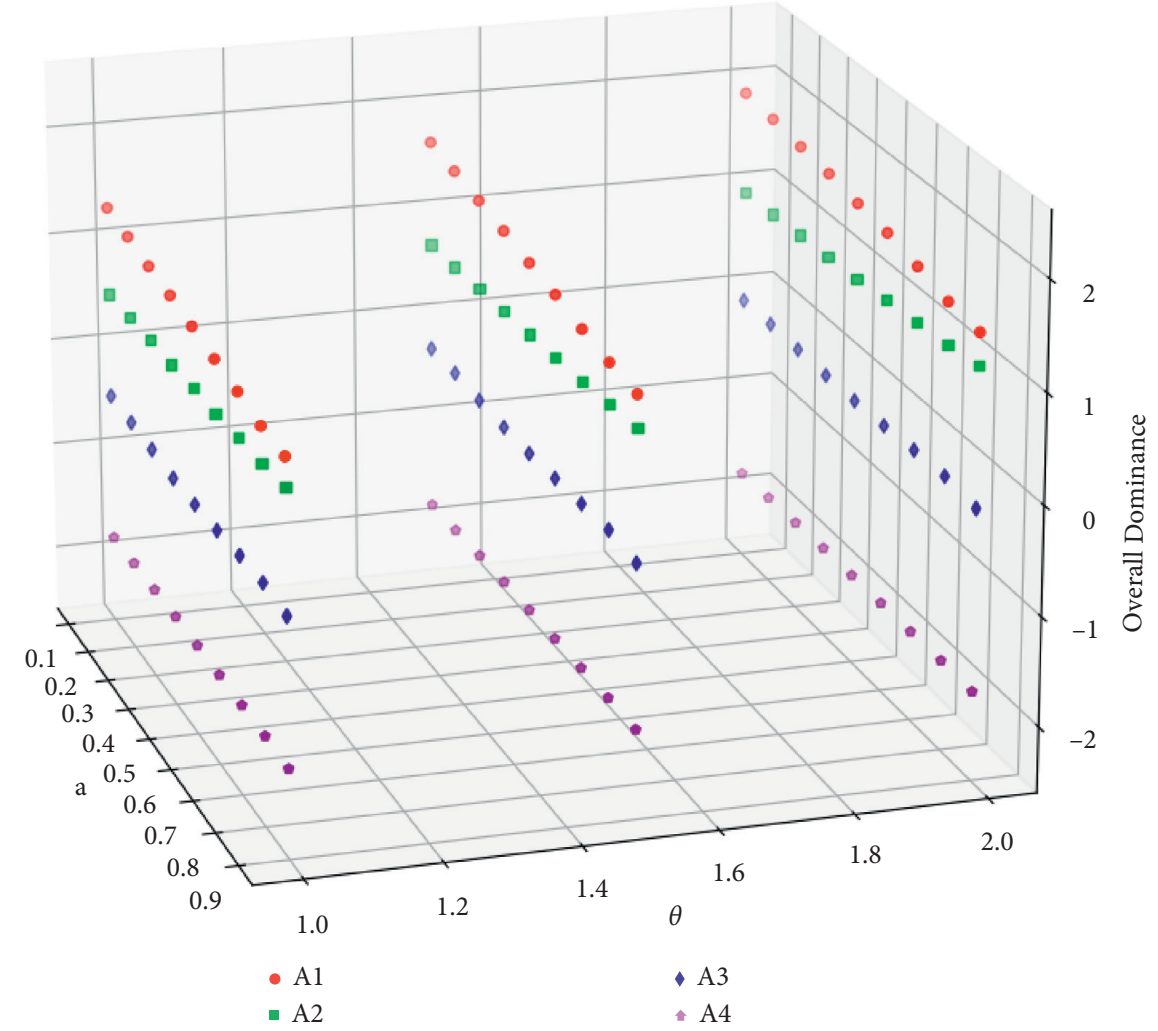

FIgURE 6: The pictorial expressions of the dominance values with different values of $\alpha$ and $\theta$ in case of $q=3$.

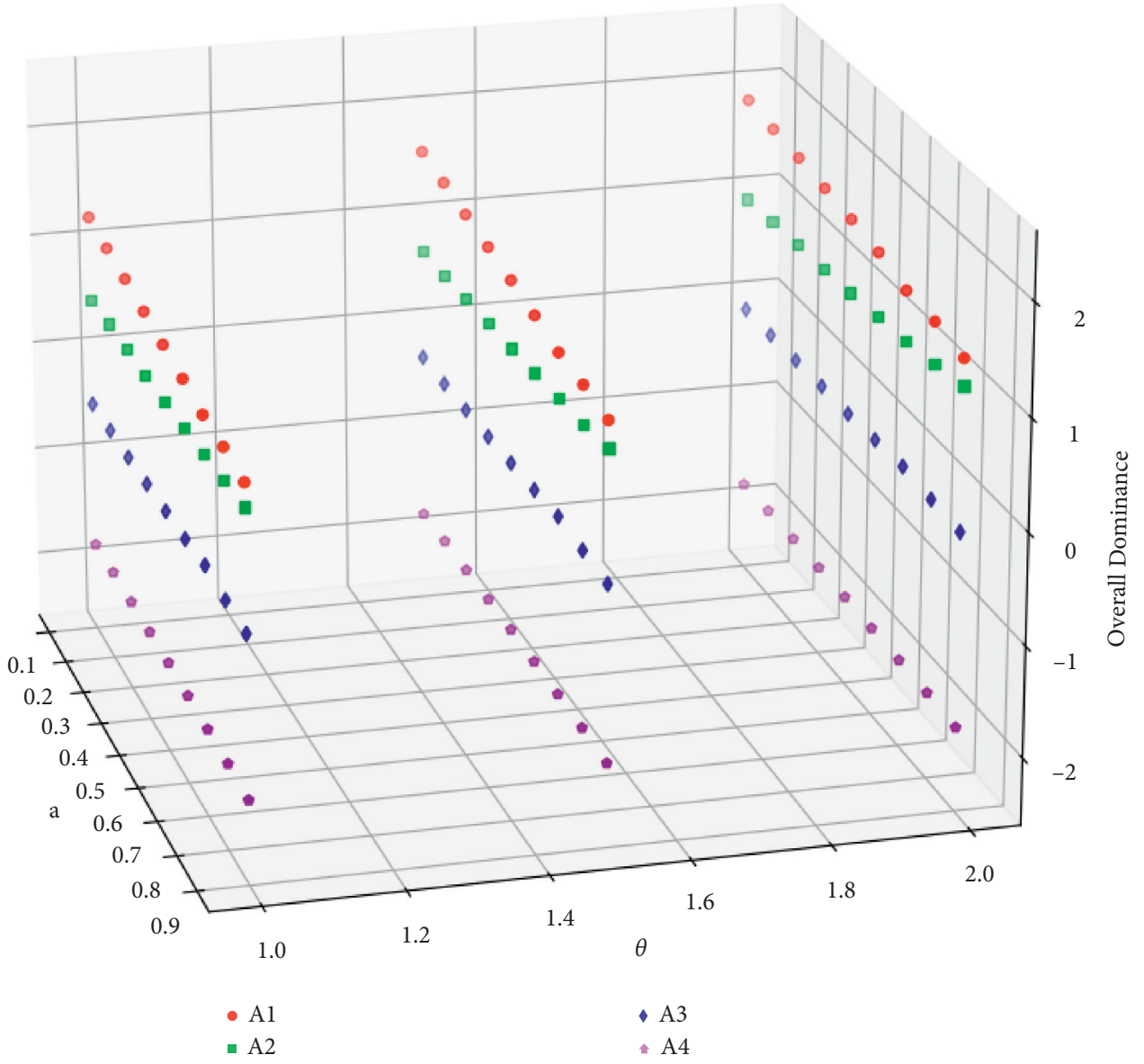

FIgURE 7: The pictorial expressions of the dominance values with different values of $\alpha$ and $\theta$ in case of $q=4$. 
TABLE 9: The comparison analysis results with different methods.

Methods Calculation results Ranking orders of risk levels

The modified TODIM proposed in this paper
The overall dominances $[1.16,0.66,-0.39,-1.75]$

Distances between collective value and PIS $[0.27,0.28,0.35$,
Method proposed in [33] based on PTFHA

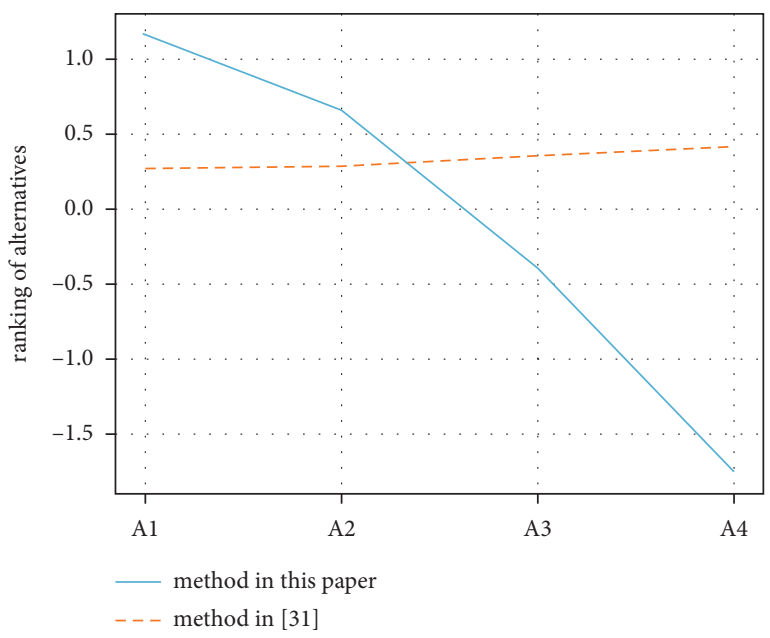

FIGURE 8: The comparison analysis results with different methods.

By equation (52), we can get the individual overall preference Pythagorean trapezoidal fuzzy values as follows:

$$
\begin{aligned}
& \widehat{z}_{1}^{(1)}=(0.29,0.43,0.55,0.69 ; 0.81,0.25), \\
& \widehat{z}_{1}^{(2)}=(0.48,0.59,0.72,0.82 ; 0.81,0.39), \\
& \widehat{z}_{1}^{(3)}=(0.23,0.33,0.50,0.61 ; 0.62,0.36), \\
& \widehat{z}_{1}^{(4)}=(0.20,0.31,0.46,0.58 ; 0.37,0.59), \\
& \widehat{z}_{2}^{(1)}=(0.23,0.33,0.50,0.65 ; 0.78,0.22), \\
& \widehat{z}_{2}^{(2)}=(0.40,0.50,0.61,0.81 ; 0.68,0.28), \\
& \widehat{z}_{2}^{(3)}=(0.19,0.37,0.51,0.70 ; 0.54,0.40), \\
& \widehat{z}_{2}^{(4)}=(0.22,0.36,0.52,0.65 ; 0.45,0.58), \\
& \widehat{z}_{3}^{(1)}=(0.26,0.39,0.55,0.69 ; 0.75,0.25), \\
& \hat{z}_{3}^{(2)}=(0.30,0.40,0.54,0.65 ; 0.62,0.16), \\
& \hat{z}_{3}^{(3)}=(0.30,0.42,0.56,0.67 ; 0.49,0.24), \\
& \widehat{z}_{3}^{(4)}=(0.22,0.32,0.46,0.58 ; 0.38,0.46), \\
& \hat{z}_{4}^{(1)}=(0.45,0.62,0.80,0.90 ; 0.77,0.35), \\
& \widehat{z}_{4}^{(2)}=(0.26,0.40,0.50,0.66 ; 0.69,0.30), \\
& \hat{z}_{4}^{(3)}=(0.26,0.45,0.61,0.72 ; 0.47,0.45), \\
& \hat{z}_{4}^{(4)}=(0.16,0.33,0.43,0.62 ; 0.27,0.53) .
\end{aligned}
$$

Next, the following PTFHA operator developed in [33] is used to obtain the collective overall preference Pythagorean trapezoidal fuzzy values: 0.42]

$$
A_{1}>A_{2}>A_{3}>A_{4}
$$$$
A_{1}>A_{2}>A_{3}>A_{4}
$$

The following results are obtained:

$$
\begin{aligned}
& \widehat{z}_{1}=(0.29,0.42,0.58,0.72 ; 0.77,0.25), \\
& \widehat{z}_{2}=(0.35,0.46,0.58,0.73 ; 0.69,0.25), \\
& \widehat{z}_{3}=(0.24,0.40,0.54,0.68 ; 0.53,0.34), \\
& \widehat{z}_{4}=(0.21,0.33,0.48,0.61 ; 0.39,0.53) .
\end{aligned}
$$

And, the Pythagorean trapezoidal fuzzy distance between each risk level and the positive-ideal solution $\widehat{z}^{+}=$ $(1,1,1,1 ; 1,0)$ is as below:

$$
\begin{aligned}
& \hat{d}\left(\widehat{z}^{1}, \widehat{z}^{+}\right)=0.27, \\
& \hat{d}\left(\widehat{z}^{2}, \widehat{z}^{+}\right)=0.28, \\
& \widehat{d}\left(\widehat{z}^{3}, \widehat{z}^{+}\right)=0.35, \\
& \widehat{d}\left(\widehat{z}^{4}, \widehat{z}^{+}\right)=0.42 .
\end{aligned}
$$

It is easy to see that

$$
\widehat{d}\left(\widehat{z}^{1}, \widehat{z}^{+}\right)<\widehat{d}\left(\widehat{z}^{2}, \widehat{z}^{+}\right)<\widehat{d}\left(\widehat{z}^{3}, \widehat{z}^{+}\right)<\widehat{d}\left(\widehat{z}^{4}, \widehat{z}^{+}\right) .
$$

According to the idea of the developed method in [33], the closer distance to the positive-ideal solution $\widehat{z}^{+}$is, the better the solution is. Thus, the ranking order of risk levels is $A_{1}>A_{2}>A_{3}>A_{4}$.

Furthermore, the comparison analysis results obtained by the developed modified TODIM method and the method proposed in [33] are shown in Table 9 and Figure 8. It can be easily found that the best alternative according to the method proposed in this paper and the method developed in [33] is the same, i.e., $A_{1}$. From the calculation results of our developed method, it can be found that $A_{1}$ has obvious advantages over other risk levels, which means that the method proposed in this paper can highlight the optimal solution among all risk levels in group decision-making. 
Comparing with the method introduced in [33], the biggest advantage of the developed method is that it can take into account the psychological behavior of decision makers and can well solve decision-making problems with risk characteristics. Meanwhile, the compromise distance proposed in this paper takes both positive-ideal solution and negativeideal solution into consideration, which is more objective and reasonable comparing with the method introduced in [33] that only considers the influence of the positive-ideal solution $\widehat{z}^{+}=(1,1,1,1 ; 1,0)$.

\section{Conclusion}

In this paper, we have introduced the concept of $q$-ROTrFNs and developed the basic operation of $q$-ROTrFNs. On the basis of the developed score function and exact function of $q$-ROTrFNs, we have proposed a new ranking method for $q$ ROTrFNs. To integrate the individual decision matrices into the collective decision matrix, we have proposed a new $q$ ROTrFWA operator and investigated its properties. Next, a new distance measure-based compromise approach has been proposed to determine the relative dominance degree of alternatives. Then, we have developed a new q-rung orthopair trapezoidal fuzzy modified TODIM group decision-making method. The psychological state of decision makers in actual decision-making process can be taken full into account in the modified TODIM method. This method can handle decision-making problems with risk characteristics well and make the decision-making results more reasonable and authentic. Finally, the modified TODIM method is verified and analyzed by the illustrating example, and the obtained optimal solution is consistent with the results obtained by [33]. At the same time, it has been verified that the different values of $q, \alpha$, and $\theta$ do not affect the ranking results. This analysis also proves the rationality and effectiveness of the group method proposed in this paper.

However, there are still some limitations in the study. First, the proposed GDM method is restricted to the $q$-rung orthopair trapezoidal fuzzy environment and relies on the experience and knowledge of the decision maker to obtain ranking of alternatives. When the group of decision makers is too large or the external environment influences strongly, the GDM method may not be able to adapt to the changes in the subjective attitude of the decision makers in time, which leads to deviations in results. Second, consistency and consensus are paid more and more attention in MAGDM [34]. However, the consensus among decision makers has not been studied in depth in this study; individual judgment is the main basis for obtaining results. As a result, it makes sense to further build the consensus model and integrate it into the proposed method. It is interesting to build the consistency and consensus model for MAGDM problems with $q$-ROTrFNs considering decision makers' risk expectation in the future, such as research on consistency and consensus model of $q$-rung orthopair trapezoidal fuzzy preference relations based on prospect theory. Meanwhile, with the development of big data and artificial intelligence, it needs to fully consider the psychological behavior of the decision maker in order to obtain accurate decision-making results in the actual decision-making process. The fusion of the developed decision-making method in this paper, machine learning, and big data are among our research directions in the future, and the developed group decisionmaking method in a heterogeneous environment is also the focus of our future research.

\section{Data Availability}

All data used to support the findings of the study are included within the article.

\section{Conflicts of Interest}

The authors declare that they have no conflicts of interest.

\section{Acknowledgments}

This work was supported in part by National Science Foundation of China, under Grants 71901112, 71661010, and 61363075, Department of Shenzhen Local Science and Technology Development, under Grant 2021Szvup052, Natural Science Foundation of Jiangxi Province of China, under Grants 20202BAB202006 and 20202BAA208012, Department of Science and Technology of Jiangxi Province of China, under Grants 20161BBG7007 and KJLD13031, Department of Education of Jiangxi Province of China, under Grants GJJ180270 and GJJ160432, and Social Science Planning Project of Jiangxi Province of China, under Grant 19GL16.

\section{References}

[1] L. A. Zadeh, "Fuzzy sets," Information and Control, vol. 8, no. 3, pp. 338-353, 1965.

[2] K. T. Atanassov, "Intuitionistic fuzzy sets," Fuzzy Sets and Systems, vol. 20, no. 1, pp. 87-96, 1986.

[3] R. R. Yager, "Pythagorean membership grades in multicriteria decision making," IEEE Transactions on Fuzzy Systems, vol. 22, no. 4, pp. 958-965, 2013.

[4] X. Zhang and Z. Xu, "Extension of TOPSIS to multiple criteria decision making with pythagorean fuzzy sets," International Journal of Intelligent Systems, vol. 29, no. 12, pp. 1061-1078, 2014.

[5] X. Zhang, "Multicriteria pythagorean fuzzy decision analysis: a hierarchical QUALIFLEX approach with the closeness index-based ranking methods," Information Sciences, vol. 330, pp. 104-124, 2016.

[6] X. Zhang, "Pythagorean fuzzy clustering analysis: a hierarchical clustering algorithm with the ratio index-based ranking methods," International Journal of Intelligent Systems, vol. 33, no. 9, pp. 1798-1822, 2018.

[7] X. Zhang, "A novel approach based on similarity measure for pythagorean fuzzy multiple criteria group decision making," International Journal of Intelligent Systems, vol. 31, no. 6, pp. 593-611, 2016.

[8] R. R. Yager, "Generalized orthopair fuzzy sets," IEEE Transactions on Fuzzy Systems, vol. 25, no. 5, pp. 1222-1230, 2016. 
[9] R. R. Yager and N. Alajlan, "Approximate reasoning with generalized orthopair fuzzy sets," Information Fusion, vol. 38, pp. 65-73, 2017.

[10] J. Gao, Z. Liang, and Z. Xu, “Additive integrals of q-rung orthopair fuzzy functions," IEEE Transactions on Cybernetics, vol. 50, no. 10, pp. 4406-4419, 2019.

[11] J. Gao, Z. Liang, J. Shang, and Z. Xu, "Continuities, derivatives, and differentials of $q$-rung orthopair fuzzy functions," IEEE Transactions on Fuzzy Systems, vol. 27, no. 8, pp. 1687-1699, 2018.

[12] X. Shu, Z. Ai, Z. Xu, and J. Ye, "Integrations of q-rung orthopair fuzzy continuous information," IEEE Transactions on Fuzzy Systems, vol. 27, no. 10, pp. 1974-1985, 2019.

[13] P. Liu, S. M. Chen, and P. Wang, "Multiple-attribute group decision-making based on $q$-rung orthopair fuzzy power maclaurin symmetric mean operators," IEEE Transactions on Systems, Man, and Cybernetics: Systems, vol. 50, no. 10, pp. 3741-3756, 2018.

[14] G. Wei, H. Gao, and Y. Wei, "Some q-rung orthopair fuzzy heronian mean operators in multiple attribute decision making," International Journal of Intelligent Systems, vol. 33, no. 7, pp. 1426-1458, 2018.

[15] P. Liu and P. Wang, "Multiple-attribute decision-making based on archimedean bonferroni operators of q-rung orthopair fuzzy numbers," IEEE Transactions on Fuzzy Systems, vol. 27, no. 5, pp. 834-848, 2018.

[16] H. Garg and S.-M. Chen, "Multiattribute group decision making based on neutrality aggregation operators of $q$-rung orthopair fuzzy sets," Information Sciences, vol. 517, pp. 427-447, 2020.

[17] Y. Yang, Z. S. Chen, R. M. Rodríguez, W. Pedrycz, and K.-S. Chin, "Novel fusion strategies for continuous intervalvalued $q$-rung orthopair fuzzy information: a case study in quality assessment of SmartWatch appearance design," International Journal of Machine Learning and Cybernetics, pp. 1-24, 2021.

[18] X. Peng and L. Liu, "Information measures for q-rung orthopair fuzzy sets," International Journal of Intelligent Systems, vol. 34, no. 8, pp. 1795-1834, 2019.

[19] H. Liao, H. Zhang, C. Zhang, X. Wu, A. Mardani, and A. AlBarakat, "A q-rung orthopair fuzzy GLDS method for investment evaluation of BE angel capital in China," Technological and Economic Development of Economy, vol. 26, no. 1, pp. 103-134, 2020.

[20] D. Banerjee, B. Dutta, D. Guha, and L. Martínez, "SMAAQUALIFLEX methodology to handle multicriteria decisionmaking problems based on $q$-rung fuzzy set with hierarchical structure of criteria using bipolar choquet integral," International Journal of Intelligent Systems, vol. 35, no. 3, pp. 401-431, 2020.

[21] L. Gomes and M. Lima, "TODIM: basics and application to multicriteria ranking of projects with environmental impacts," Foundations of Computing and Decision Sciences, vol. 16, no. 4, pp. 113-127, 1992.

[22] L. F. A. M. Gomes, M. A. S. Machado, and L. A. D. Rangel, "Behavioral multi-criteria decision analysis: the TODIM method with criteria interactions," Annals of Operations Research, vol. 211, no. 1, pp. 531-548, 2013.

[23] Z.-P. Fan, X. Zhang, F.-D. Chen, and Y. Liu, "Extended TODIM method for hybrid multiple attribute decision making problems," Knowledge-Based Systems, vol. 42, pp. $40-48,2013$.
[24] R. Lourenzutti and R. A. Krohling, "A study of TODIM in a intuitionistic fuzzy and random environment," Expert Systems with Applications, vol. 40, no. 16, pp. 6459-6468, 2013.

[25] X. Zhang and Z. Xu, "The TODIM analysis approach based on novel measured functions under hesitant fuzzy environment," Knowledge-Based Systems, vol. 61, pp. 48-58, 2014.

[26] J. Qin, X. Liu, and W. Pedrycz, "An extended TODIM multicriteria group decision making method for green supplier selection in interval type-2 fuzzy environment," European Journal of Operational Research, vol. 258, no. 2, pp. 626-638, 2017.

[27] P. Ren, Z. Xu, and X. Gou, "Pythagorean fuzzy TODIM approach to multi-criteria decision making," Applied Soft Computing, vol. 42, pp. 246-259, 2016.

[28] P. Liu, M. Shen, F. Teng, B. Zhu, L. Rong, and Y. Geng, "Double hierarchy hesitant fuzzy linguistic entropy-based TODIM approach using evidential theory," Information Sciences, vol. 547, pp. 223-243, 2021.

[29] W. Yu, Z. Zhang, Q. Zhong, and L. Sun, "Extended TODIM for multi-criteria group decision making based on unbalanced hesitant fuzzy linguistic term sets," Computers \& Industrial Engineering, vol. 114, pp. 316-328, 2017.

[30] Q. Wu, X. Liu, J. Qin, W.-Z. Wang, and L. Zhou, “A linguistic distribution behavioral multi-criteria group decision making model integrating extended generalized TODIM and quantum decision theory," Applied Soft Computing, vol. 98, Article ID 106757, 2021.

[31] C. Wei, Z. Ren, and R. M. Rodríguez, "A hesitant fuzzy linguistic TODIM method based on a score function," International Journal of Computational Intelligence Systems, vol. 8, no. 4, pp. 701-712, 2015.

[32] X. Tian, W. Li, L. Liu, and G. Kou, "Development of TODIM with different types of fuzzy sets: a state-of the-art survey," Applied Soft Computing, vol. 111, Article ID 107661, 2021.

[33] M. Shakeel, S. Abduulah, M. Shahzad, T. Mahmood, and N. Siddiqui, "Averaging aggregation operators with pythagorean trapezoidal fuzzy numbers and their application to group decision making," Journal of Intelligent \& Fuzzy Systems, vol. 36, no. 2, pp. 1899-1915, 2019.

[34] Z. Zhang, Z. Li, and Y. Gao, "Consensus reaching for group decision making with multi-granular unbalanced linguistic information: a bounded confidence and minimum adjustment-based approach," Information Fusion, vol. 74, pp. 96-110, 2021. 